\title{
One-dimensional ballistic transport with FLAPW Wannier functions
}

\author{
Björn Hardrat, ${ }^{1, *}$ Neng-Ping Wang, ${ }^{2}$ Frank Freimuth, ${ }^{3}$ Yuriy Mokrousov, ${ }^{3}$ and Stefan Heinze ${ }^{1}$ \\ ${ }^{1}$ Institut für Theoretische Physik und Astrophysik, Christian-Albrechts-Universität zu Kiel, Leibnizstrasse 15, D-24098 Kiel, Germany \\ ${ }^{2}$ Physics Department, Ningbo University, Fenghua Road 818, 315211 Ningbo, People's Republic of China \\ ${ }^{3}$ Peter Grünberg Institut and Institute for Advanced Simulation, Forschungszentrum Jülich and JARA, D-52425 Jülich, Germany
}

(Received 23 January 2012; published 5 June 2012)

\begin{abstract}
We present an implementation of the ballistic Landauer-Büttiker transport scheme in one-dimensional systems based on density functional theory calculations within the full-potential linearized augmented plane-wave (FLAPW) method. In order to calculate the conductance within the Green's function method, we map the electronic structure from the extended states of the FLAPW calculation to Wannier functions, which constitute a minimal localized basis set. Our approach benefits from the high accuracy of the underlying FLAPW calculations, allowing us to address the complex interplay of structure, magnetism, and spin-orbit coupling and is ideally suited to study spin-dependent electronic transport in one-dimensional magnetic nanostructures. To illustrate our approach, we study ballistic electron transport in nonmagnetic Pt monowires with a single stretched bond including spin-orbit coupling, and in ferromagnetic Co monowires with different collinear magnetic alignment of the electrodes with the purpose of analyzing the magnetoresistance when going from tunneling to the contact regime. We further investigate spin-orbit scattering due to an impurity atom. We consider two configurations: a Co atom in a Pt monowire and vice versa. In both cases, the spin-orbit induced band mixing leads to a change of the conductance upon switching the magnetization direction from along the chain axis to perpendicular to it. The main contribution stems from ballistic spin scattering for the magnetic Co impurity in the nonmagnetic Pt monowire, and for the Pt scatterer in the magnetic Co monowire from the band formed from states with $d_{x y}$ and $d_{x^{2}-y^{2}}$ orbital symmetry. We quantify this effect by calculating the ballistic anisotropic magnetoresistance, which displays values up to as much as $7 \%$ for ballistic spin scattering and gigantic values of around $100 \%$ for the $\mathrm{Pt}$ impurity in the Co wire. In addition, we show that the presence of a scatterer can reduce as well as increase the ballistic anisotropic magnetoresistance.
\end{abstract}

DOI: 10.1103/PhysRevB.85.245412

PACS number(s): 73.23.Ad, 31.15.A-, 72.10.-d, 73.63.-b

\section{INTRODUCTION}

With the possibility to perform transport measurements on nanoscale down to atomic-scale junctions using mechanically controllable break junctions ${ }^{1}$ or scanning tunneling microscopy, ${ }^{2-7}$ various fundamental questions on electron transport as well as practical problems concerning device functionality have arisen. With shrinking system size, the junctions have become considerably smaller than the mean-free path of a transmitted electron, reaching the ballistic transport regime. In this regime, various effects such as the geometric arrangement of the atoms, the chemical composition, the magnetic order, vibrations, correlation effects, or the magnetic anisotropy can play an important role due to the reduced coordination number of the participating atoms. In the context of spin-dependent transport, for example, there is a strong interest in understanding how the spin-valve effect scales to systems of atomic or molecular scale., ${ }^{7,8}$ In nanoscale junctions, new transport effects can also arise such as the ballistic anisotropic magnetoresistance (BAMR). ${ }^{9,10}$ In order to successfully address such issues, a theoretical description needs to properly take into account the electronic structure of the system, which is typically obtained by first-principles methods based on density functional theory (DFT). The central experimental quantity is the measured current versus bias voltage (I-V curve) or, at small bias voltages, the conductance.

The theoretical method most frequently applied to describe quantum transport in such systems is the Landauer-Büttiker approach in which the junction is divided into a central scattering region and two leads in thermal equilibrium with contact reser- voirs (Fig. 1), resulting in the famous Landauer conductance formula. ${ }^{11}$ Basically, two different groups of techniques have been developed to solve the transport problem: wave-function based and Green's function (GF) based methods, which are equivalent in case of noninteracting charge carriers. ${ }^{12}$ Among the wave-function based methods, the transmission through such a quasi-one-dimensional (1D) system can be calculated by means of the transfer matrix method, ${ }^{13-17}$ solving the Lippmann-Schwinger equation, ${ }^{18,19}$ or by wave-function matching. ${ }^{20}$ The GF methods are usually based on Keldysh,

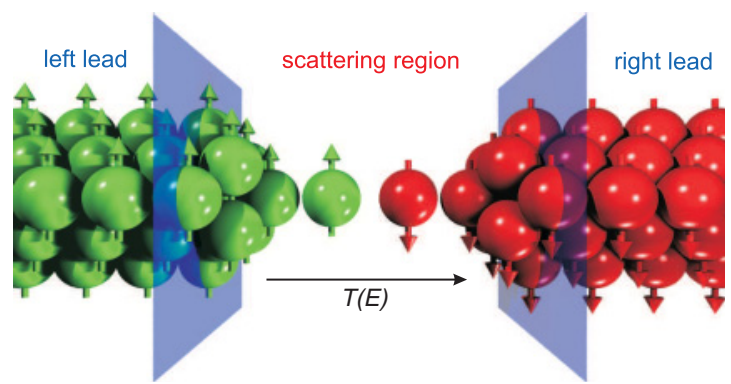

FIG. 1. (Color online) Typical geometry of a ballistic transport calculation, consisting of three different regions (left lead/scattering region/right lead). Charge carriers with energy $E$ are transmitted through a scattering region with a transmission probability $T(E)$ from the left lead to the right lead. The blue planes separate the leads from the scattering region. While the semi-infinite leads resemble the electronic structure of a periodic system, the scattering region includes the scatterer as well as the lead-scatterer contact region. 
Kadanoff, and Baym's nonequilibrium Green's functions (NEGF). ${ }^{21,22}$ Beyond the standard noninteracting electron approach, there has been work incorporating, e.g., inelastic scattering on vibrations ${ }^{23,24}$ or treating correlation effects through self-energies. ${ }^{25}$ An alternative way of calculating quantum transport is by using the Kubo approach as formulated by Baranger and Stone, ${ }^{26}$ relating the current to the dynamical polarization. $^{27,28}$

Based on these three general approaches, all codes differ in the way the electronic structure is described. In the first implementations based on density functional theory, the electrodes were treated as jellium which were coupled to the scattering region. ${ }^{15,17,18}$ Large systems up to devices can be described using semiempirical tight-binding methods for the electronic structure, ${ }^{13,14,19,29,30}$ while approaches using DFT for both the description of the electrodes via self-energies and the scattering region promise the highest accuracy. ${ }^{27,28,31-44}$

Among these implementations, various DFT methods have been applied. Transport codes based on Green's functions rely on a localized basis set, limiting this approach to basis sets of numerical orbitals such as Gaussians, ${ }^{34,42}$ localized orbitals, ${ }^{32,33,35,38,45}$ or wavelets. ${ }^{36}$ The application of flexible and accurate plane-wave DFT methods for transport calculations is usually realized in connection with the scattering approach for the conductance. ${ }^{46,47}$ Alternatively, the efficient GF method for the transport calculation can be used if the extended states in the plane-wave expansion are mapped onto maximally localized Wannier functions ${ }^{48}$ (MLWFs). This approach combines plane-wave calculations with the use of a minimal basis set suitable for quantum transport calculations. ${ }^{36,37,43,49,50}$ When one is dealing with lowdimensional systems and subtle band-structure effects such as spin-orbit coupling, the accuracy of electronic-structure description becomes crucial. Therefore, the application of a highly precise all-electron full-potential linearized augmented plane-wave code is desirable. To our knowledge, no such DFT transport scheme has been reported, and only a few codes allow to incorporate spin-orbit coupling. ${ }^{47,51-53}$

In this paper, we present a method to calculate transport through 1D nanoscale structures following the LandauerBüttiker approach. The underlying electronic structure of the studied system is obtained from DFT within the $1 \mathrm{D}$ version ${ }^{54}$ of the full-potential linearized augmented plane-wave method (FLAPW), as implemented within the FLEUR code. ${ }^{55}$ The 1D FLAPW method is specifically tailored to treat 1D structures avoiding supercell calculations: the periodicity is explicitly taken into account only along the nanostructure's axis ( $z$ axis in the following), while the wave functions in the vacuum surrounding the system are forced to obey an exponential decay. ${ }^{54}$ Since the FLAPW wave functions are intrinsically delocalized in real space, we perform a mapping of the electronic structure of the system onto a set of localized Wannier functions (WFs), which allows us to solve the transport problem in real space efficiently. The WFs obtained from the FLAPW calculation (FLAPW WFs) (Refs. 56 and 57) provide a minimal localized basis set which describes the $a b$ initio electronic bands within a certain energy window with high accuracy and allows us to efficiently compute the nonequilibrium Green's function (NEGF) of the system needed to determine its transmission function $T(E)$. We use and compare two different sets of WFs, namely, the maximally localized Wannier functions, ${ }^{48}$ which are uniquely defined by fulfilling the condition of maximal localization in real space, and the so-called first-shot Wannier functions (FSWFs), ${ }^{56}$ being much easier to obtain computationally and, although nonunique, still capable of describing the transport properties of a system correctly in many cases. A special approximation we include in our transport scheme is the so-called "locking technique," which allows us to use separately calculated leads and scattering regions and to combine those into one quantum transport calculation, achieving an accurate treatment of leads and scattering region at reduced computational cost.

As a first application, we have calculated the electronic structure and the ballistic transport properties of a nonmagnetic Pt monowire with a single stretched bond in the middle of the chain, which acts as a source of scattering. For this rather simple system, we demonstrate the quality of our MLWFs and FSWFs, the locking technique to obtain the Hamiltonian of the open system, and show the possibility of decomposing the transmission function in terms of orbital symmetry. We further investigate the influence of spin-orbit-coupling (SOC) on the transmission of the $\mathrm{Pt}$ wire. We find a substantial change of the conductance of one quantum of conductance at the Fermi level for a perfect wire due to the strength of SOC in $5 d$-transition metals such as Pt.

In order to include the effect of large spin polarization, we have chosen a ferromagnetic Co monowire with a single stretched bond, a prototypical magnetic system, and calculate the magnetoresistance from the conductance in a parallel and antiparallel alignment of the Co electrodes. We obtain a rapid decrease of the magnetoresistance with the separation between the two Co monowires, which is due to the fast decay of transmission from the highly spin-polarized localized states of $d_{x z, y z}$ and $d_{x y, x^{2}-y^{2}}$ symmetry.

Finally, we have studied scattering from a single impurity atom in a monowire due to SOC. We have chosen two configurations: (i) a nonmagnetic $\mathrm{Pt}$ atom in a ferromagnetic Co wire and (ii) a magnetic Co atom in a nonmagnetic Pt wire. In both cases, we have compared the conductance obtained in the scalar-relativistic approximation and upon including SOC. We find a strong influence of SOC on the transmission due to the induced splitting of bands. In addition, the conductance depends sensitively on the magnetization direction in the system being either along the wire axis or perpendicular to it. While in case (ii) the resulting ballistic anisotropic magnetoresistance displays values of $7 \%$ due to spin-orbit interaction mediated scattering into both spin channels for the symmetry-breaking out-of-chain quantization axis, in case (i) the values of BAMR reach as much as $100 \%$, reflecting the giant value of the ballistic anisotropic magnetoresistance of the pure Co chain.

The paper is organized as follows. In Sec. II, we describe the theoretical basis of our approach to calculate the conductance and introduce the key quantities. In particular, the Green's function method is applied to obtain the transmission function and the conductance. The mapping of the electronic structure from the FLAPW method to a localized basis set is accomplished via Wannier functions. The construction of the Hamiltonian for the open quantum system is described. In Sec. III, we present the first applications of our transport 
code to several typical systems of interest. We begin with the conductance for a nonmagnetic Pt wire with a single broken bond and study the transmission as a function of bond length and upon including spin-orbit coupling. Then, the magnetoresistance of Co monowires with a single elongated bond is discussed. Finally, the effect of spin-orbit scattering is illustrated by two examples: a Pt monowire with a single magnetic $\mathrm{Co}$ atom and a Co monowire with a single $\mathrm{Pt}$ atom. A summary and conclusions are given in Sec. VI.

\section{METHOD}

\section{A. General transport problem}

We describe the transport properties of the system within the Landauer-Büttiker approach, dividing it into three different regions: two semi-infinite leads (left $L$ and right $R$ ) and the scattering region $(S)$, which includes the actual scatterer as well as the lead-scatterer contact region, in which the effect of the scatterer on the properties of the leads ideally decays such that their electronic structure can be considered perfect and unperturbed inside the $L$ and $R$ regions. Assuming that the interaction between the left and right leads can be neglected, the tight-binding Hamiltonian of our system corresponding to such a structural division has the following form:

$$
\mathbf{H}=\left(\begin{array}{lll}
\mathbf{H}_{L} & \mathbf{H}_{L S}^{\dagger} & 0 \\
\mathbf{H}_{L S} & \mathbf{H}_{S} & \mathbf{H}_{S R} \\
0 & \mathbf{H}_{S R}^{\dagger} & \mathbf{H}_{R}
\end{array}\right)
$$

where $\mathbf{H}_{L / R}$ is the semi-infinite Hamiltonian of the left/right lead, while $\mathbf{H}_{L S / S R}$ describes the coupling of the scattering region to the leads and $\mathbf{H}_{S}$ is the Hamiltonian of the scattering region. Due to the semi-infiniteness of the leads, the dimension of the Hamiltonian (1) is infinite, which presents a conceptual computational problem. An efficient method to deal with that, applicable to any system of the type depicted in Fig. 1, which can be described with a real-space tight-binding Hamiltonian of the type of Eq. (1), has been developed. ${ }^{58,59}$ This method is based on the nonequilibrium Green's function formalism, which treats the scattering region and the semi-infinite leads on equal footing, describes extractions, reinjections, and excitations of electrons in the system and solves the problem of the semi-infinite leads by introducing finite-dimensional self-energies $\Sigma g_{L / R}$, which include the true lead's effect on the scattering process. Within the NEGF formalism, the system is described by means of the retarded Green's function

$$
\mathbf{G}(E)=[(E+i \epsilon) \mathbf{I}-\mathbf{H}]^{-1},
$$

where I denotes the unity matrix of the dimension of $\mathbf{H}$. By neglecting at first the coupling of the leads to the scattering region and regarding just the first few layers of the leads which are actually interacting with the scattering region, it is possible to replace the leads' Green's function by their surface Green's functions $\mathbf{g}_{L / R}(E) .{ }^{58,59}$ This can be derived by rewriting the lead's Hamiltonian in a block-diagonal form using square matrices $\mathbf{h}_{L / R}$ and $\mathbf{h}_{L L / R R}$ of the same dimension as the surface Green's function:

$$
\mathbf{H}_{L}=\left(\begin{array}{cccc}
\ddots & & & \mathbf{0} \\
& \mathbf{h}_{L} & \mathbf{h}_{L L}^{\dagger} & \\
& \mathbf{h}_{L L} & \mathbf{h}_{L} & \mathbf{h}_{L L}^{\dagger} \\
\mathbf{0} & & \mathbf{h}_{L L} & \mathbf{h}_{L}
\end{array}\right) .
$$

Based on this description of the leads, the surface Green's function $\mathbf{g}_{L / R}(E)$ can be determined iteratively, starting from

$$
\mathbf{g}_{L / R}^{[0]}(E)=\left[(E+i \epsilon) \mathbf{I}_{L / R}-\mathbf{h}_{L / R}\right]^{-1},
$$

with $\mathbf{g}_{L / R}^{[0]}(E)$ being a square matrix with the dimension of interacting orbitals at the leads' surfaces. The expression (4) can be converged to the surface Green's function by recursively incorporating the interlayer interaction submatrices $\mathbf{h}_{L L / R R}$ with an efficient recursive scheme. ${ }^{60}$

By reintroducing the coupling of the scattering region to the leads as a perturbation to the system, the Green's function $\mathbf{G}_{S}(E)$ of the scattering region can be obtained from the unperturbed Green's function of the scattering region by the Dyson equation

$$
\mathbf{G}_{S}(E)=\left[E \mathbf{I}_{S}-\mathbf{H}_{S}-\mathbf{H}_{L S}^{\dagger} \mathbf{g}_{L} \mathbf{H}_{L S}-\mathbf{H}_{S R}^{\dagger} \mathbf{g}_{R} \mathbf{H}_{S R}\right]^{-1} .
$$

The whole effect of the semi-infinite leads on the conductor can be then expressed by the leads' self-energies $\Sigma_{L / R}(E)$, which incorporate the surface Green's function $\mathbf{g}_{L / R}(E)$ and the now finite-sized coupling matrices $\mathbf{H}_{L S / S R}$ adapted to the size of the surface Green's functions:

$$
\boldsymbol{\Sigma}_{L / R}(E)=\mathbf{H}_{L S / S R}^{\dagger} \mathbf{g}_{L / R}(E) \mathbf{H}_{L S / S R} .
$$

The self-energies are obviously finite-sized matrices of the dimension of $\mathbf{H}_{S}$. The self-energies are related to the broadening matrices $\Gamma$ :

$$
\Gamma_{L / R}(E)=i\left[\Sigma_{L / R}(E)-\Sigma_{L / R}^{\dagger}(E)\right],
$$

which describe the effect of broadening of the states in the scattering region caused by the presence of the leads as well as the transfer rates of charge carriers from the leads into the scattering region. The incorporation of the non-Hermitian self-energies changes the nature of the description from the static steady-state picture of the open system to a dynamic transport scheme, responding to an incoming charge carrier with the energy $E$. Based on these quantities, the transmission function $T(E)$, describing the probability of charge carriers originating from one lead to be transmitted to the other lead, can be expressed in the following way:

$$
T(E)=\operatorname{Tr}\left[\mathbf{G}_{S}(E) \boldsymbol{\Gamma}_{L}(E) \mathbf{G}_{S}^{\dagger}(E) \boldsymbol{\Gamma}_{R}(E)\right] .
$$

The current, being a natural observable in a quantum transport measurement, can then be calculated from the Landauer formula

$$
I=\frac{e}{h} \int d E T(E)\left[f_{L}(E)-f_{R}(E)\right],
$$

where $f_{L / R}$ are the occupation functions of the leads. The expression for the conductance then reads as

$$
G(E)=\frac{e^{2}}{h} T(E) .
$$


In the case of perfect transmission $T(E)=1$, this results in the well-known conductance quantum

$$
G_{0}=\frac{2 e^{2}}{h} .
$$

for a single, spin-degenerate band.

\section{B. From FLAPW states to localized Wannier functions}

The aim of the approach introduced here is to combine the accuracy and speed of state-of-the-art DFT electronic-structure calculations based on the one-dimensional version of the FLAPW method as implemented in the FLEUR code, ${ }^{54}$ and the capability of the NEGF formalism described above to treat the whole transport problem in an efficient way. In particular, for transport phenomena driven by magnetism or spin-orbit coupling, a precise description of the electronic structure is necessary. Typical systems currently under scrutiny in experiment include geometries with a low coordination number which favors magnetism and gives rise to strong SOC due to unquenching of the orbital moment. ${ }^{2}$

The major problem in combining a LAPW or a planewave-based electronic-structure method with the real-space transport schemes lies in the fact that normally several hundreds of delocalized basis functions per atom are used in such codes in order to achieve the required accuracy. In our implementation, we use the machinery of Wannier functions, constructed out of FLAPW wave functions, ${ }^{56}$ which proved to be an efficient connection between the two, conceptually independent, computational methods. The main advantage in such a "link" can be attributed to two factors: (i) using the gauge freedom of Wannier functions, they can be enforced to be rather localized in real space, and (ii) an "exact" mapping of the $a b$ initio Hamiltonian onto a tight-binding representation with WFs as a localized orthonormal basis set can be achieved. ${ }^{61}$

Having at hand the converged Bloch wave functions $\psi_{m \mathbf{k}}$ for a set of bands $m \leqslant M$ calculated on a uniform mesh of $\mathcal{N} k$ points, the orthonormal set of Wannier functions can be obtained via the following transformation ${ }^{62}$ :

$$
\left|W_{\mathbf{R} n}\right\rangle=\frac{1}{\mathcal{N}} \sum_{\mathbf{k}} e^{-i \mathbf{k} \cdot \mathbf{R}} \sum_{m=1}^{M} U_{m n}^{\mathbf{k}}\left|\psi_{\mathbf{k} m}\right\rangle,
$$

where the number of WFs $N$ should be smaller than or equal to $M$. The gauge freedom of WFs manifests itself in that the matrices $U_{m n}^{\mathbf{k}}$ (in the following, $\mathbf{U}$ matrices) can in principle be arbitrary. In the case when $N=M$ and the group of bands from which we are extracting the WFs is isolated from other bands, the $\mathbf{U}$ matrices are unitary at each $k$ point. Imposing the constraint of maximal localization of WFs in real space determines the set of $\mathbf{U}$ matrices up to a common global phase, and the corresponding set of WFs is called the maximally localized Wannier functions. ${ }^{48}$ For the whole procedure of maximal localization, we use the WANNIER90 code. ${ }^{63}$

The criterion for the localization of WFs is the smallness of their spread. ${ }^{48}$ The process of the spread minimization constitutes an iterative process at the end of which the $\mathbf{U}$ matrices corresponding to the MLWFs are obtained. This minimization procedure requires as a starting point a certain initial guess for the set of the MLWFs. In order to construct this set, one chooses certain localized orbitals $\left|g_{n}\right\rangle$, which are projected onto the subspace of wave functions $\left|\psi_{\mathbf{k} m}\right\rangle$ :

$$
\left|\phi_{\mathbf{k} n}\right\rangle=\sum_{m}\left|\psi_{\mathbf{k} m}\right\rangle\left\langle\psi_{\mathbf{k} m} \mid g_{n}\right\rangle,
$$

and then orthonormalized

$$
\left|\tilde{\psi}_{\mathbf{k} n}\right\rangle=\sum_{m}\left(\left(\mathbf{S}^{(\mathbf{k})}\right)\right)^{-\frac{1}{2}}\left|\phi_{\mathbf{k} m}\right\rangle,
$$

with the overlap matrix $S_{m n}^{(\mathbf{k})}=\left\langle\phi_{\mathbf{k} m} \mid \phi_{\mathbf{k} n}\right\rangle$, after which the starting WFs can be generated:

$$
\left|W_{\mathbf{R} n}\right\rangle=\frac{1}{\mathcal{N}} \sum_{\mathbf{k}} e^{-i \mathbf{k} \cdot \mathbf{R}}\left|\tilde{\psi}_{\mathbf{k} n}\right\rangle
$$

This orthonormal set of Wannier orbitals we will call in the following the first-shot WFs (FSWFs).

The FSWFs are not unique in the sense that they strongly depend on the choice of the localized orbitals $g_{n}$. In many cases, however, especially when MLWFs are well localized around atoms as in the case of certain $d$ orbitals in most of transition metals and transition-metal oxides, ${ }^{64}$ the difference between the FSWFs, originated from the localized $d$ orbitals, and the corresponding MLWFs is rather small. This allows us to spare the computational time needed for the minimization of the spread, and immediately construct, e.g., the needed effective Hamiltonians in terms of FSWFs. Examples, when there is a substantial difference between the FSWFs and MLWFs, include orbitals for which the centers of the WFs do not coincide with the centers of atoms. In the following, we will analyze in detail the difference in transport properties calculated with MLWFs and FSWFs, both in the case when there is little difference between the two sets of WFs and when the difference between them is significant.

\section{Construction of the Hamiltonian in real space}

In terms of the FLAPW basis functions, the Hamiltonian can be written as

$$
\mathbf{H}_{\mathrm{FLAPW}}=\frac{1}{\mathcal{N}} \sum_{m \mathbf{k}} \varepsilon_{m}(\mathbf{k})\left|\psi_{m \mathbf{k}}\right\rangle\left\langle\psi_{m \mathbf{k}}\right|,
$$

while in terms of WFs the equivalent expression is

$$
\mathbf{H}_{\mathrm{WFs}}=\sum_{n \mathbf{R}_{1}} \sum_{n^{\prime} \mathbf{R}_{2}} H_{n, n^{\prime}}\left(\mathbf{R}_{1}-\mathbf{R}_{2}\right)\left|W_{n \mathbf{R}_{1}}\right\rangle\left\langle W_{n^{\prime} \mathbf{R}_{2}}\right|,
$$

where

$$
H_{n, n^{\prime}}\left(\mathbf{R}_{1}-\mathbf{R}_{2}\right)=\left\langle W_{n \mathbf{R}_{1}}\left|\mathbf{H}_{\mathrm{FLAPW}}\right| W_{n^{\prime} \mathbf{R}_{2}}\right\rangle
$$

are the hopping integrals between the $n$ and $n^{\prime}$ Wannier orbitals at sites $\mathbf{R}_{1}$ and $\mathbf{R}_{2}$. By substituting Eq. (16) into Eq. (18), we find

$$
H_{n, n^{\prime}}\left(\mathbf{R}_{1}-\mathbf{R}_{2}\right)=\frac{1}{\mathcal{N}} \sum_{m, \mathbf{k}} \varepsilon_{m}(\mathbf{k})\left\langle W_{n \mathbf{R}_{1}} \mid \psi_{m \mathbf{k}}\right\rangle\left\langle\psi_{m \mathbf{k}} \mid W_{n^{\prime} \mathbf{R}_{2}}\right\rangle .
$$

Thus, the real-space representation of the Hamiltonian in terms of localized Wannier functions can be derived from the knowledge of the eigenvalues and wave functions of the system. In respect to WFs, for efficient evaluation of Eq. (19), only knowledge of the $\mathbf{U}$ matrices is required. ${ }^{56}$ The 


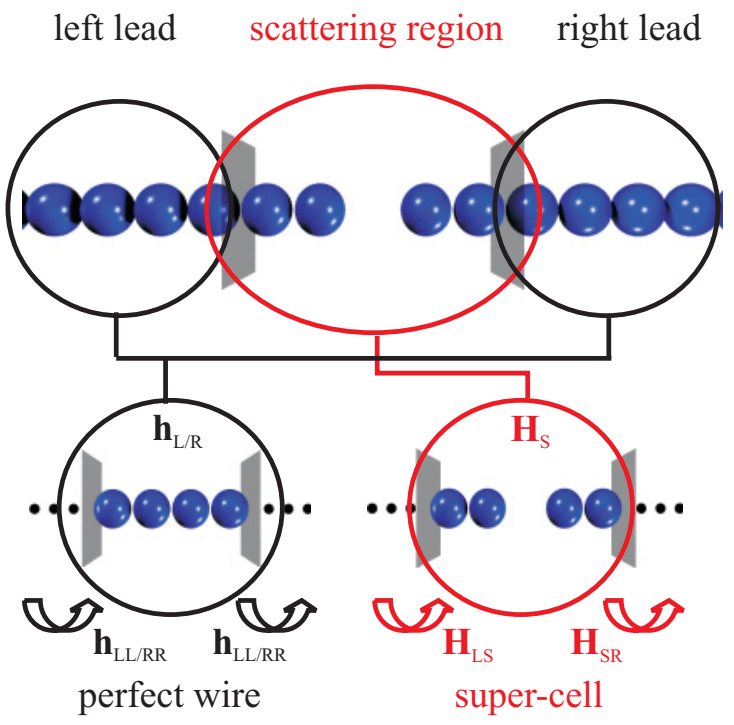

FIG. 2. (Color online) Schematic sketch of a ballistic transport calculation based on a WFs tight-binding Hamiltonian. The leads are described by perfect wires to exclude spurious deviations from their exact electronic structure. Their semi-infinite structure is constructed from the Hamiltonians of principal layers $\mathbf{h}_{L / R}$ and the interaction matrices $\mathbf{h}_{L L / R R}$ between two principal layers. The scattering region is described by the Hamiltonian $\mathbf{H}_{S}$ and coupled to the leads by the interaction matrices $\mathbf{H}_{L R / S R}$, extracted from a supercell calculation. The supercell has to be large enough to reproduce the lead-scatterer contact with desired accuracy, usually larger than sketched here.

correspondence in the eigenspectrum between the constructed Hamiltonian in terms of WFs and the Hamiltonian in terms of eigenfunctions is exact on the grid of $k$ points used for the WFs construction, and for this reason the set of WFs is sometimes referred to as exact basis set, or, the tight-binding basis set of $a b$ initio accuracy. (This is only valid within the frozen inner window of disentangled systems. ${ }^{65}$ )

According to Eqs. (4) to (8), in order to calculate the transmission function, only the $\mathbf{h}_{L / R}, \mathbf{h}_{L L / R R}, \mathbf{H}_{L S / S R}$, and $\mathbf{H}_{S}$ parts of the Hamiltonian are needed. Given a FLEUR electronic-structure calculation, it is necessary to construct these parts of the Hamiltonian from the resulting WFs hopping elements [Eq. (19)]. We focus on the correct treatment of the scattering region (see Fig. 2).

After determination of the atoms belonging to the scattering region, it is possible to write down the preliminary result for $\mathbf{H}_{S}$, based on Eq. (19),

$$
\mathbf{H}_{S}=\sum_{i, n} \sum_{j, m} H_{n, m}\left(\mathbf{R}_{i}-\mathbf{R}_{j}\right)\left|W_{n \mathbf{R}_{i}}\right\rangle\left\langle W_{m \mathbf{R}_{j}}\right|,
$$

where $i$ and $j$ determine the atom and $n$ and $m$ the inherent WFs.

Due to the real-space decay of the WFs, the corresponding hopping matrix elements $H_{n, n^{\prime}}\left(\mathbf{R}_{i}-\mathbf{R}_{j}\right)$ also decay as the distance in real space between the Wannier functions $\left|\mathbf{R}_{i}-\mathbf{R}_{j}\right|$ is increasing. For an efficient use of the real-space WFs Hamiltonian within the transport scheme described above, it is necessary to keep its matrix elements only up to a certain number of nearest neighbors (NN), setting the rest of the elements to zero. As a result of this procedure, the Hamiltonian matrix becomes sparse, which allows for a computationally inexpensive treatment. For a given number of NN, the quality of the sparse Hamiltonian depends on the degree of localization of the WFs. Here, by quality of the Hamiltonian, we mean the correspondence between its eigenvalue spectrum to that obtained from $a b$ initio, or, in the sense of transport, how well converged the transmission function $T(E)$ is with respect to the number of NN. In this respect, in the following we compare and analyze the results obtained with MLWFs and FSWFs, which display different localization properties.

One way to deal with the exponential decay in Eq. (20) would be to manually eliminate all matrix elements beyond a certain NN. We propose here a flexible scheme, minimizing this effort by dividing the scattering region into principal layers $\mathbf{h}_{l}, l=1, \ldots, s$, and interaction matrices $\mathbf{h}_{l, l+1}$ between neighboring layers:

$$
\mathbf{H}_{S}=\left(\begin{array}{cccc}
\mathbf{h}_{1} & \mathbf{h}_{12}^{\dagger} & & \mathbf{0} \\
\mathbf{h}_{12} & \ddots & \ddots & \\
& \ddots & \mathbf{h}_{s-1} & \mathbf{h}_{(s-1) s}^{\dagger} \\
\mathbf{0} & & \mathbf{h}_{(s-1) s} & \mathbf{h}_{s}
\end{array}\right) .
$$

The submatrices are set up as Eq. (20). For the onsite matrices $\mathbf{h}_{l}$, the indices $i$ and $j$ are restricted to atoms from the given layer $l$. For the interaction matrices $\mathbf{h}_{l, l+1}$, the index $i$ is restricted to atoms from layer $l$ and the index $j$ to atoms of the neighboring layer $l+1$.

While still capable of describing the system in terms of Eq. (20) (with $s=1$ ), the principal layers can optimally contain the number of atoms effectively interacting, reducing the number of neglected hoppings. Typically, these principal layers are chosen to contain the same number of atoms as the principal layers of the leads, resulting in the same approximation in terms of $\mathrm{NN}$ for both regions and thereby avoids inconsistencies in the transport calculations. Furthermore, this scheme allows possible future extensions such as, e.g., a combination of separately calculated scatterers into one scattering region.

Knowing the Hamiltonian $\mathbf{H}_{S}$ of the scattering region, it is necessary to determine the coupling of the scattering region to the leads. Since the Hamiltonians of both leads and the scattering region are partitioned into principal layers [see Eqs. (3) and (21)], we only need to find the hopping elements between the adjacent layers. Interactions between non-neighboring layers are neglected by construction. The nonzero elements of $\mathbf{H}_{L S}$ can now be extracted from the supercell calculation (see Fig. 2) as

$$
\mathbf{H}_{L S}=\sum_{i, n} \sum_{j, m} H_{n, m}\left(\mathbf{R}_{i}-\mathbf{R}_{j}\right)\left|W_{n \mathbf{R}_{i}}\right\rangle\left\langle W_{m \mathbf{R}_{j}}\right|,
$$

where the index $i$ runs over the atoms of the principal layer 1 of $\mathbf{H}_{S}$ and the index $j$ runs over the principal layer of the left lead. $\mathbf{H}_{S R}$ can be constructed analogously. To prevent a significant systematical error, it is necessary to make the original supercell large enough to screen an unphysical interunit cell interaction.

Finally, only the Hamiltonians for the leads are missing. Ideally, the calculated unit cell should be large enough in order to reproduce the properties of the bulk material far away from the scatterer and thus, the lead Hamiltonian can be extracted 
directly from the supercell calculations in a straightforward manner. Owing to the significant computational burden, it is, however, hardly feasible to apply this approach to large and complex systems while keeping at the same time the accuracy necessary to capture the main energy scales of the phenomena studied. The technique we use to overcome this problem, particularly prominent for the FLAPW method with its complicated basis set, is discussed in the following section.

Up to now, no comments have been made concerning the way magnetic systems and the effect of SOC are treated. For magnetic systems, the majority and minority spin channels can be regarded separately, resulting in two independent calculations of the transmission function for spin-up and spin-down channels. In the presence of SOC, the whole methodology holds considering that both spin channels have to be treated together, thus resulting in twice the number of WFs used simultaneously to solve the transport problem.

\section{Locking technique}

The accurate treatment of the leads within the approach described above constitutes a considerable challenge. Taken from a self-consistent supercell electronic-structure calculation as they are, the submatrices $\mathbf{h}_{L / R}$ and $\mathbf{h}_{L L / R R}$ will contain deviations from "ideal"-lead matrix elements in a large vicinity of the scattering region. While some of these deviations are definitely physical in their origin due to a large decay length of 1D charge perturbations caused by the scatterer, the rest of them will be a spurious artifact of the supercell approach owing to the fact that the leads as calculated are not intrinsically semi-infinite. This presents a considerable problem in particular when the leads have to be described with Hamiltonians beyond the 1st NN. In this case, to describe the semi-infinite leads precisely one would have to go to huge supercells so that the $A$ atoms in the supercell describing the lead would be exactly identical, with $A$ being the number of atoms in one principal layer [see Eq. (3)]. We found that condition impossible to achieve for nontrivial systems. Another approach of constructing a lead beyond 1st $\mathrm{NN}$ artificially from the outmost atoms of the scattering region by periodically expanding it is flawed, too, due to the unknown unperturbed hopping matrix elements beyond $1 \mathrm{st}$ NN. This is a serious problem since the lead has to be described as precisely as possible to prevent a huge systematic error.

The basic idea to work around this problem is as simple as effective, namely, matching the supercell hopping matrix elements to those of the true leads. Within this so-called "locking" technique, the leads are replaced by the perfect wires, providing correct self-energies and Fermi levels of the true infinite periodic system, while the supercell size is chosen large enough to describe the lead-scatterer interface region sufficiently well (see Fig. 2). In our transport approach, this means that different parts of the Hamiltonian [Eqs. (20) and (22)] are extracted from two different DFT calculations ${ }^{66-68}$ : the $\mathbf{H}_{S}$ and $\mathbf{H}_{L S / S R}$ coupling matrices are taken from the supercell calculation describing the scattering region, while the $\mathbf{h}_{L / R}$ and $\mathbf{h}_{L L / R R}$ submatrices [needed in Eq. (3)] are taken from the calculation for the perfect leads. $\mathbf{h}_{L / R}$ and $\mathbf{h}_{L L / R R}$ can be determined similar to the principal layers $\mathbf{h}_{l}$ and $\mathbf{h}_{l, l+1}$ of $\mathbf{H}_{S}$ [Eqs. (20) and (21)] with the principal layer $l$ and the neighboring identical layer $l+1$. To achieve matching Fermi levels for lead and supercell calculations, it is additionally necessary to align the diagonal elements of the matrices $\mathbf{h}_{L / R}$ [Eq. (3)] and $\mathbf{H}_{S}$ [Eq. (21)].

\section{Pt MONOWIRES}

In the following sections, we present a few instructive applications which illustrate the quality and possibilities of our FLAPW WF based approach to obtain the conductance in onedimensional magnetic systems within the Landauer coherent transport method. In this section, we focus on Pt monowires, which possess a single stretched bond that acts as a scattering potential for electrons. Starting from the construction of the WFs and the tight-binding-like Hamiltonian, we discuss the transmission function and its decomposition in eigenchannels. Our results further demonstrate the applicability of the locking technique described above. Finally, we include spin-orbit coupling in our calculations and show that the obtained transmission compares well with that calculated based on the scattering approach in combination with a pseudopotential method for the electronic structure. ${ }^{47}$

In order to calculate the conductance within the approach described in the previous sections, we need to perform two separate DFT calculations and subsequent Wannierizations for every system: (i) a calculation for the semi-infinite electrode and (ii) a supercell calculation which includes the scattering center. From the latter, we determine the hopping matrix elements for the coupling to the leads. For the monowires considered in the following, the Hamiltonian of the semiinfinite electrode can be obtained from a calculation with one atom in the unit cell. For the scattering region, we have used supercells of different sizes as described in the computational details section in the Appendix.

\section{A. Band structure and hoppings}

Before proceeding into the discussion of the transmission, it is insightful to examine the localization properties of typical MLWFs and FSWFs which we use for our transport calculations. While the unique MLWFs are rather well localized in real space, this is not necessarily the case for the FSWFs, which strongly depend on the choice of the initial orbitals. If the trial orbitals do not differ very much from the final result of the localization procedure, the difference in spread between the MLWFs and the FSWFs can be small.

For transition-metal monowires, this is the case for the localized $d$ orbitals of $\Delta_{3}$ symmetry $\left(d_{x z}\right.$ and $\left.d_{y z}\right)$ and of $\Delta_{4}$ symmetry $\left(d_{x y}\right.$ and $\left.d_{x^{2}-y^{2}}\right)$. Taking an infinite periodic $\mathrm{Pt}$ monoatomic chain with an interatomic spacing of $4.48 \mathrm{bohr}$ as an example, we calculate the spreads of the $\Delta_{3}$ and $\Delta_{4}$ MLWFs to be 3.70 and 2.22 bohr $^{2}$, respectively. The calculated spreads of the FSWFs, constructed with solutions of the radial equation for the actual potential obtained from the firstprinciples calculation, ${ }^{56}$ are indistinguishable from the former.

The situation is completely different, however, for the FSWFs constructed from the $s$ - and $d_{z^{2}}$-like trial orbitals. In this case, the difference in spread between the resulting FSWFs and the $\Delta_{1}$-like MLWFs is remarkable. While values 


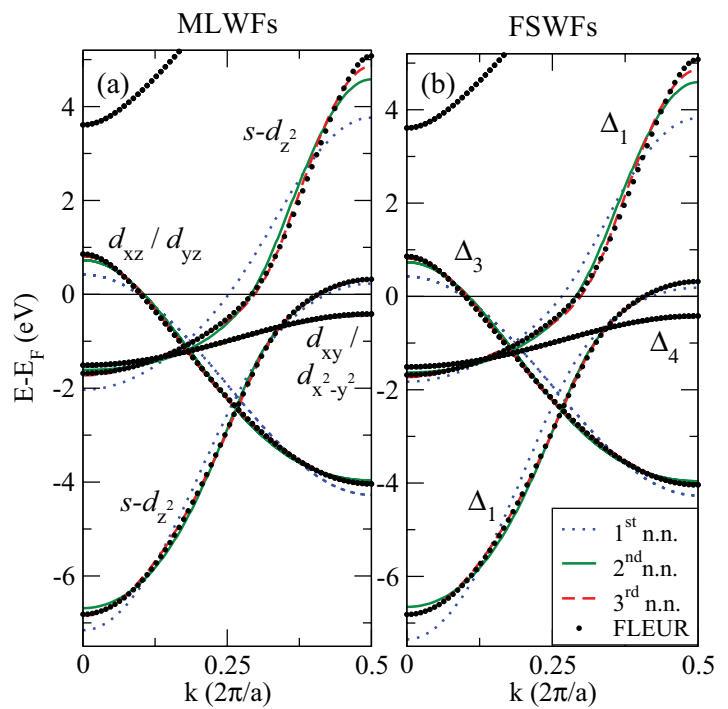

FIG. 3. (Color online) Comparison of the Pt monowire band structure in SR approximation calculated within DFT (big black dots) and obtained from the WF Hamiltonian based on (a) MLWFs and (b) FSWFs considering a limited number of nearest neighbors. In (a) the orbital character of the states is given and in (b) the bands are denoted according to their symmetry with respect to the chain geometry.

of 2.89 and $6.20 \mathrm{bohr}^{2}$ are obtained for the spread of $d_{z^{2}}$-like and $s$-like MLWFs, respectively, the corresponding values constitute 55.78 and 319.44 bohr $^{2}$ for FSWFs. This indicates that the MLWFs differ significantly from the trial functions.

The reason for the rather large spreads of the FSWFs can be found by comparing the FSWF centers to the MLWFs centers. In the case of MLWFs, the centers of the $s$-like WFs are located between the atoms, forming covalent bridgelike Wannier functions. Such Wannier functions are hard to construct directly from the atom-centered trial orbitals. The FSWFs constructed from the $s$ - and $d_{z^{2}}$-like trial orbitals are, in contrast, located on the atoms, which causes a significantly larger spread. ${ }^{69}$

In principle, all Hamiltonians obtained by mapping to Wannier functions which include the hopping matrix elements between all WFs are equivalent. This equivalency is lifted, however, if we consider only a limited number of neighbors to set up our tight-binding-like Hamiltonian. In Fig. 3, the Pt monowire band structure based on the FLAPW calculation and Slater-Koster interpolations of the band structure based on MLWFs and FSWFs are compared. The trial orbitals for the FSWFs are in this case chosen to be $s$ - and $d$-like orbitals and centered on each atom. While in first-NN approximation the interpolated band structures differ between the MLWFs and FSWFs approach, especially in the bandwidth of the more delocalized $s$ and $d_{z^{2}}$ orbitals, already in the second-NN approximation both WFs basis sets describe the FLAPW band structure equally well. By further increasing the considered number of neighbors to the third-NN approximation, the accuracy of the description increases with respect to the $s$ bandwidth. However, the most important part with respect to transport properties is the band structure in the vicinity of the Fermi level, which does not improve significantly. For the more
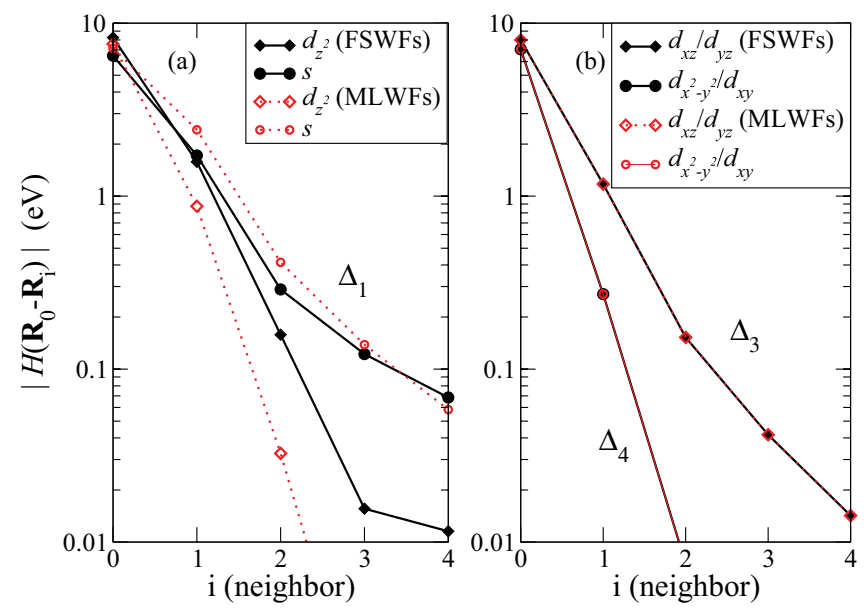

FIG. 4. (Color online) Real-space hopping integrals between orbitals of the same type $\left|H\left(\mathbf{R}_{i}-\mathbf{R}_{0}\right)\right|$ as a function of the $\mathrm{NN}$ for a $\mathrm{Pt}$ monowire on a logarithmic scale. The hoppings were calculated both with MLWFs (open red symbols) and FSWFs (closed black symbols) for (a) $s$ and $d_{z^{2}}$ orbitals, and for (b) $d_{x y}, d_{x^{2}-y^{2}}, d_{x z}$, and $d_{y z}$ orbitals.

localized $d_{x y}, d_{x^{2}-y^{2}}, d_{x z}$, and $d_{y z}$ orbitals, even the first-NN description is sufficient as seen in the band structure and also from the hopping matrix elements as seen in Fig. 4.

At least for a rather simple system such as a perfect $\mathrm{Pt}$ monowire, the localization procedure used to obtain MLWFs obviously does not influence the localized $d$ orbitals mentioned above. Only the $s$ and $d_{z^{2}}$ states are affected, but the decay of the hopping integrals is exponential irrespective of the description (FSWFs or MLWFs). For systems more complicated than a Pt monowire, the initial choice of trial orbitals may not be straightforward. In such a case, the localization procedure to obtain MLWFs significantly improves the accuracy of the calculation, while for simpler systems where more intuitive choices of orbitals can be made, FSWFs may be sufficient. An example that both descriptions indeed lead to very similar results with respect to transport calculations is shown below for a Pt monowire with one elongated bond. Note that the FSWFs make the construction of the transport Hamiltonian, as discussed in Sec. IIC, much more simple, especially for systems with a more complex electronic structure.

\section{B. Transmission: Scalar-relativistic case}

With the aid of the Pt monowire DFT calculations and the construction of WFs and the Hamiltonian from the hopping matrix elements, it is now possible to calculate the conductance based on the Green's function method. We start by considering the quality of the locking technique. For this purpose, we compare the results for a rather small 6-atom-supercell calculation for the scattering region with a single elongated bond of $\Delta=0.72 \mathrm{bohr}$ and a calculation performed in a 12-atom supercell. The quantum conductance obtained for both cases without applying the locking technique, i.e., constructing the semi-infinite leads from the supercell calculation, is similar but differs in key details such as a sharp peak just below the Fermi energy [compare Figs. 5(a) and 5(b)]. If we replace the Hamiltonian for the leads by the one constructed from the MLWFs of a periodic Pt monowire, the result 


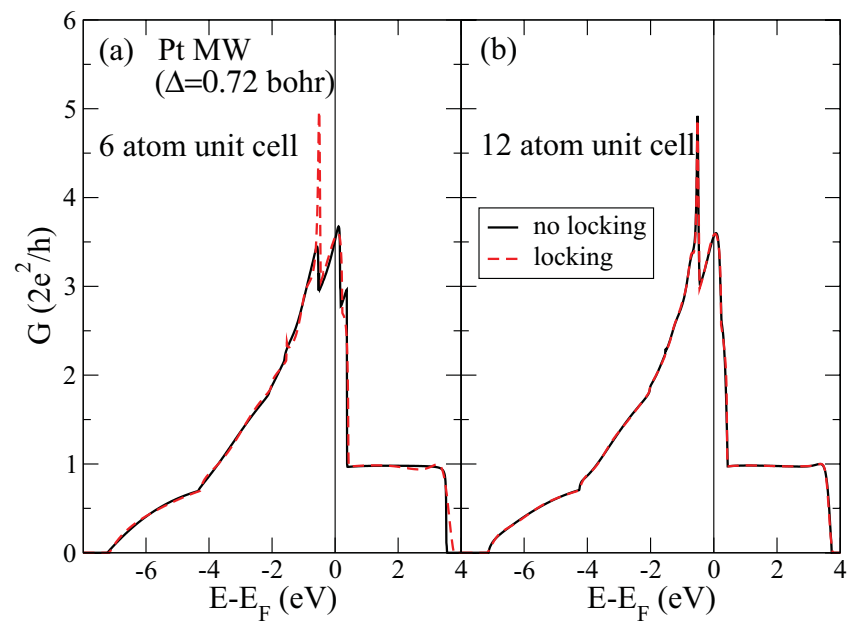

FIG. 5. (Color online) Conductance for a Pt monowire with a single bond stretched by $\Delta=0.72$ bohr using MLWFs within the nearest-neighbor approximation for the transport Hamiltonian and (a) a 6-atom supercell and (b) a 12-atom supercell for the FLAPW calculation of the scattering region. The semi-infinite leads have been described using the supercell calculation (solid lines) or using the locking technique (dashed lines), i.e., using perfect $\mathrm{Pt}$ monowires for the leads.

changes as follows: While the conductance based on the 12-atom-supercell calculation is nearly independent on how the lead was constructed, the result for the 6-atom calculation improves significantly upon using the locking technique and is almost indistinguishable from the calculation in the larger 12-atom supercell. This demonstrates the applicability and quality of the locking technique, which allows us to save a considerable amount of computational effort to calculate the ballistic transport properties.

While the previous test has been performed within the nearest-neighbor approximation for the tight-binding-like Hamiltonian, we now determine how accurate the calculated transmission function is with respect to the number of neighbors included. In Fig. 6(a), the transmission functions, calculated in first-, second-, and third-nearest-neighbor approximations and based on the 12-atom supercell for the scattering region with one stretched bond of $\Delta=0.72$ bohr are presented. The main effect which we observe upon including more neighbors is a widening of the energy range in which the conductance is nonzero as expected from the comparison of the band structure obtained in the different approximations (cf. Fig. 3). The conductance in the vicinity of the Fermi energy which is dominated by the localized $d$ states is well described already using second nearest neighbors. Using only the first nearest neighbor, on the other hand, results in an offset of the conductance above the Fermi energy which originates from a shift of the upper edge of the $\Delta_{3}$ band as seen in the band structure. Therefore, we use at least the second-nearest-neighbor approximation in the following to construct the tight-binding-like Hamiltonian.

In order to understand which states contribute to the transmission, we can decompose it with respect to the orbital symmetry of the Wannier functions. The individual transmission channels can be derived from Eq. (8) by performing the trace

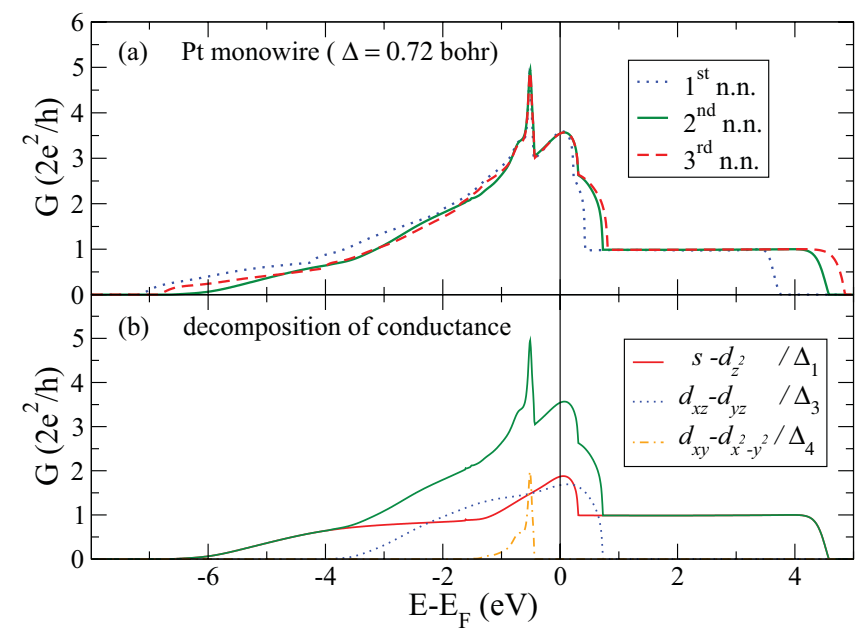

FIG. 6. (Color online) (a) Conductance for a Pt monowire with a single bond stretched by $\Delta=0.72$ bohr based on a 12-atom-supercell calculation using the first-, second-, and third-nearest-neighbor approximations for the construction of the transport Hamiltonian from MLWFs and the locking technique to attach the leads. (b) Decomposition of the total conductance (solid line) for the second-nearest-neighbor approximation into the contributions of the $s-d_{z^{2}}\left(\Delta_{1}\right)$ (solid red line), the $d_{x z}-d_{y z}\left(\Delta_{3}\right)$ (dotted blue line), and the $d_{x y}-d_{x^{2}-y^{2}}\left(\Delta_{4}\right)$ (dashed-dotted orange line) channels.

operation only over WFs within the same symmetry group. In Fig. 6(b), we see that $s-d_{z^{2}}$ states provide an almost perfectly conducting channel in a large energy range. Only far below the Fermi energy the value drops below $2 e^{2} / h$, and in the vicinity of the Fermi energy it rises due to the availability of two $\Delta_{1}$ bands (cf. the band structure in Fig. 3). The more localized $d_{x z}-d_{y z}$ states, on the other hand, possess a much smaller transmission and their contribution is localized in a small energy window. This effect is even more dramatic for the $d_{x y}-d_{x^{2}-y^{2}}$ orbitals, which show a very small overlap and hopping matrix elements leading to a sharp peak in the conductance.

Finally, we turn to the conductance of the Pt monowire as a function of the stretched bond length shown in Fig. 7. For the conductance of a perfect $\mathrm{Pt}$ wire, we find the expected step-function shape in which each band contributes with one conductance quantum $G_{0}$ per spin within its bandwidth. Upon increasing the length of a single bond in the wire, the overlap between the Wannier orbitals across the gap decreases, especially for the more localized $d$ orbitals, and as a result the transmission drops dramatically. Accordingly, only the contribution from the $s-d_{z^{2}}$ states survives at large gaps, while the sharp peak originating from the $d_{x y}-d_{x^{2}-y^{2}}$ orbitals vanishes above $\Delta=1.82 \mathrm{bohr}$. Another important result of this calculation is that the Hamiltonians obtained with MLWFs and FSWFs provide nearly the same results, i.e., the radial solutions of the FLAPW potential are evidently a reasonable choice as FSWFs trial orbitals.

\section{Transmission: Spin-orbit coupling}

For heavy transition metals such as Pt, spin-orbit coupling plays an important role and has a significant impact on the electronic structure. Evidently, the transport properties should 


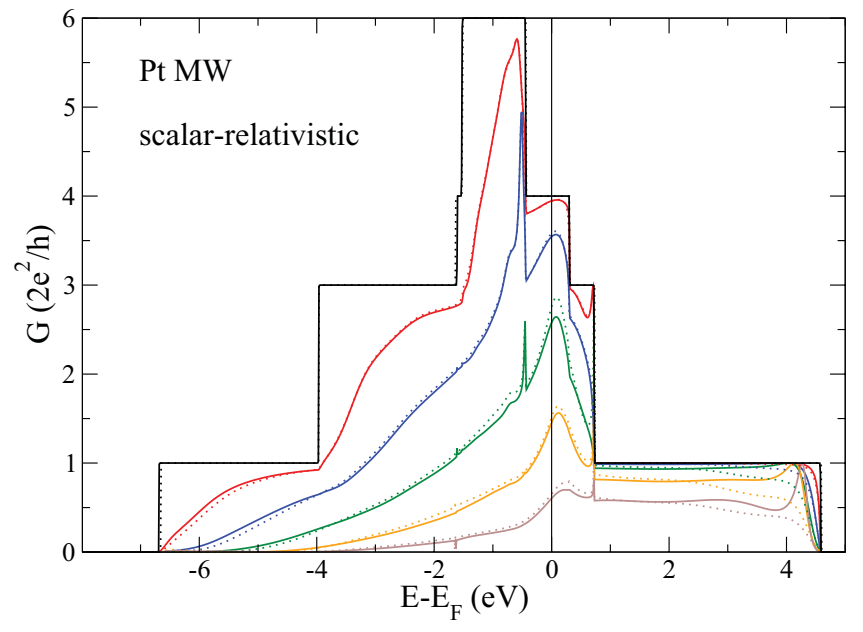

FIG. 7. (Color online) Conductance for a nonmagnetic Pt monowire in the scalar-relativistic approximation, i.e., neglecting spin-orbit coupling, with a single bond stretched by $\Delta$. The secondnearest-neighbor approximation has been used for the transport Hamiltonian. The WFs and hopping matrix elements have been constructed from a 12-atom supercell and the leads were described by the locking technique. Curves are shown for MLWFs (solid lines) and FSWFs (dotted lines) for $\Delta=0.0,0.34,0.72,1.22,1.82$, and 2.52 bohr (from left to right).

be equally affected. A suitable method to describe the quantum conductance in such systems has to be capable of treating SOC. The effect of SOC on the electronic structure, namely, the coupling of the spin quantum number $s=\frac{1}{2}$ and angular momentum quantum number $l=0,1,2, \ldots$ to the total angular momentum quantum number $j=\frac{1}{2}, \frac{3}{2}, \frac{5}{2}, \ldots$ can be seen in Fig. 8. Compared to the scalar-relativistic calculation, in which SOC is neglected (Fig. 3), the band structure including SOC

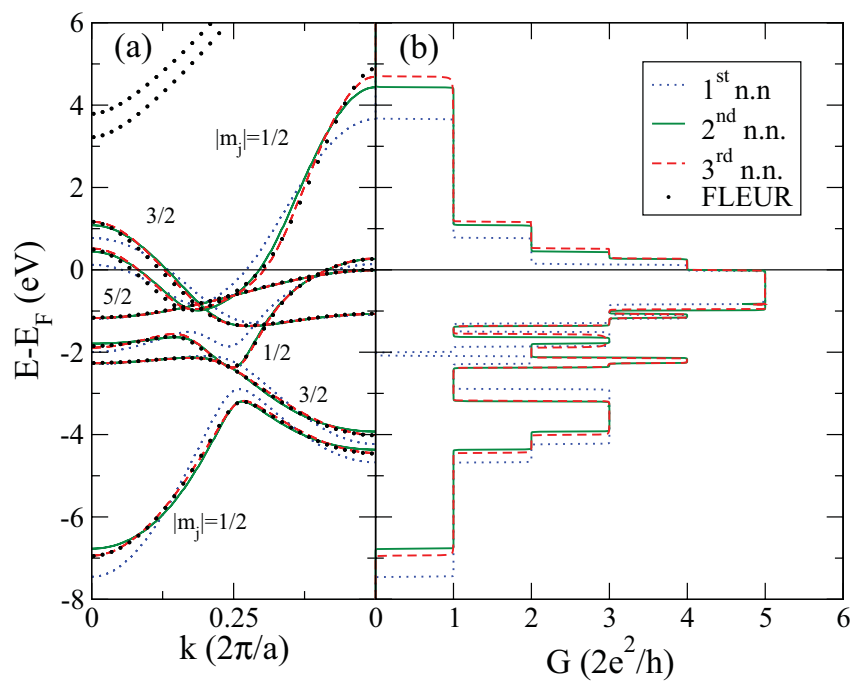

FIG. 8. (Color online) Band structure of an infinite nonmagnetic Pt monowire including spin-orbit coupling. (a) Band structure from the FLAPW calculation (big dots) and using the Hamiltonian from FSWFs within the first-, second-, and third-nearest-neighbor approximations. (b) Conductance based on FSWFs for 1st- (dotted line), 2nd- (dashed line), and 3rd- (solid line) NN approximations.

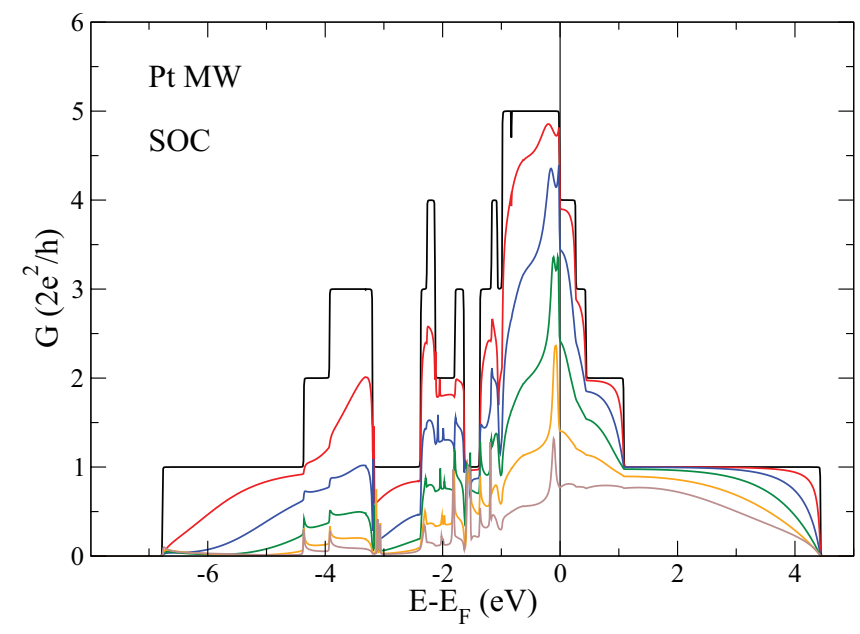

FIG. 9. (Color online) Conductance for a nonmagnetic Pt wire with a single stretched bond $d_{\mathrm{Pt}}+\Delta$ including spin-orbit coupling calculated within a 6-atom supercell and using locking to semi-infinite Pt leads based on the Hamiltonian obtained from MLWFs in the second-nearest-neighbor approximation. From left to right: one bond stretched by $\Delta=0.0,0.34,0.72,1.22,1.82$, and $2.52 \mathrm{bohr}$.

changes significantly (Fig. 8). In the chain geometry, the states are eigenfunctions to the $z$ component (chain axis) of the total angular momentum and we can classify the bands by the absolute value of $m_{j}$ as shown in Fig. 8(a). Thereby, spin-orbit coupling leads to several avoided crossings in the band structure, e.g., of a $s-d_{z^{2}}$ and $d_{x z} / d_{y z}$ band around $3 \mathrm{eV}$ below the Fermi level. With respect to the scalar-relativistic band structure, we also observe a significant shift of the $d_{x y}$ and $d_{x^{2}-y^{2}}$ bands toward the Fermi energy. As this band touches the Fermi energy at $k=\frac{\pi}{a}$, the conductance jumps from $4 G_{0}$ in the scalar-relativistic case to a value of $5 G_{0}$. This finding already demonstrates the importance of SOC for quantum transport calculations in such systems.

The general form of the conductance in presence of SOC (Fig. 9) changes significantly, too, due to the lifted degeneracies of bands with different $\left|m_{j}\right|$ values [see Fig. 8(b)]. While the conductance at the Fermi level is enhanced upon taking SOC into account, the degeneracy of the $d_{x y}$ and $d_{x^{2}-y^{2}}$ bands in the SR case leads to a higher conductance of $6 G_{0}$ below the Fermi energy. Another key difference due to SOC is the larger number of steps which appear in the conductance as a result of the anticrossings in the band structure, in particular, in the energy range of 3 to $1 \mathrm{eV}$ below the Fermi level. In Fig. 9, we also display the evolution of the conductance upon stretching a single bond in the Pt monowire. Similar to the SR case, we observe a rapid decrease of the conductance due to more localized $d$ orbitals. However, due to the spin-orbit split bands, there is a more pronounced peak structure in the conductance. In particular, we find a sharp peak just below the Fermi energy, which decays more slowly than in the SR calculation where it is located slightly lower in energy. Our calculations of the conductance are in good agreement with those obtained based on fully relativistic ultrasoft pseudopotentials and a scattering approach to obtain the conductance. ${ }^{47}$ 


\section{Co MONOWIRES}

Another important aspect in transport through nanoscale structures is the effect of spin polarization and magnetic order. Due to the reduced coordination number in nanostructures, the density of states is enhanced and, according to the Stoner model the tendency toward magnetism increases. The reduced symmetry also results in a much larger magnetocrystalline anisotropy energy (MAE) as the orbital moments become more significant. For example, freestanding and suspended chains of $4 d$ - and $5 d$-transition metals become magnetic and show giant values of the MAE, ${ }^{70,71}$ and the effect of colossal magnetic anisotropy has been reported. ${ }^{72}$ Here, we demonstrate that our method allows spin-polarized transport calculations. We consider a simple model system, i.e., a Co monowire with a single stretched bond and allow a parallel and antiparallel alignment of the magnetization on the two Co electrodes. We calculate the conductance in both configurations and determine the magnetoresistance as a function of electrode separation. The calculations in the antiferromagnetic configuration of the Co monowire can also be compared to calculations by Smogunov et al. based on the scattering approach and pseudopotentials. $^{46}$

\section{A. Magnetoresistance}

Compared to the nonmagnetic Pt band structure, the Co chain exhibits a smaller bandwidth due to more localized $3 d$ states, and a large exchange splitting (Fig. 10). The exchange splitting leads to a net spin moment in the unit cell of $2.13 \mu_{B}$. A good overall accuracy in reproducing this band structure based on FSWFs can be achieved if we go up to third-nearestneighbor hoppings. For the $d$ bands and the $s-d_{z^{2}}$ bands around the Fermi energy, even the second-nearest-neighbor approximation is sufficient. From the spin-split band structure, we expect a larger conductance in the parallel magnetization
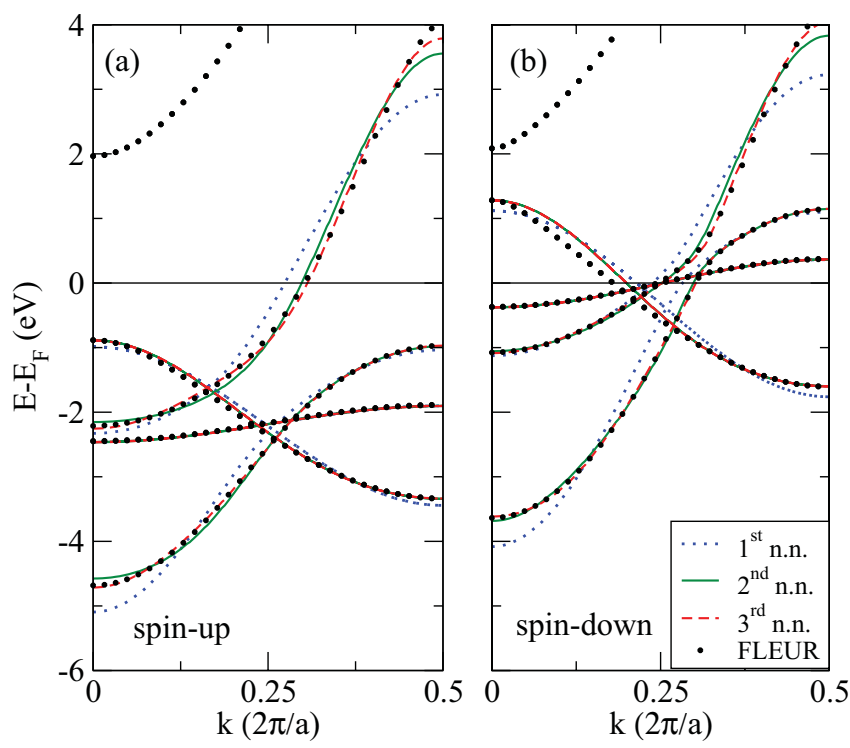

FIG. 10. (Color online) (a) Majority and (b) minority band structures for a ferromagnetic Co monowire with $d_{\mathrm{Co}}=4.15 \mathrm{bohr}$ calculated within FLAPW (big dots) and FSWFs in 1st- (dotted lines), 2nd- (dashed lines), and 3rd-NN (solid lines) approximations.

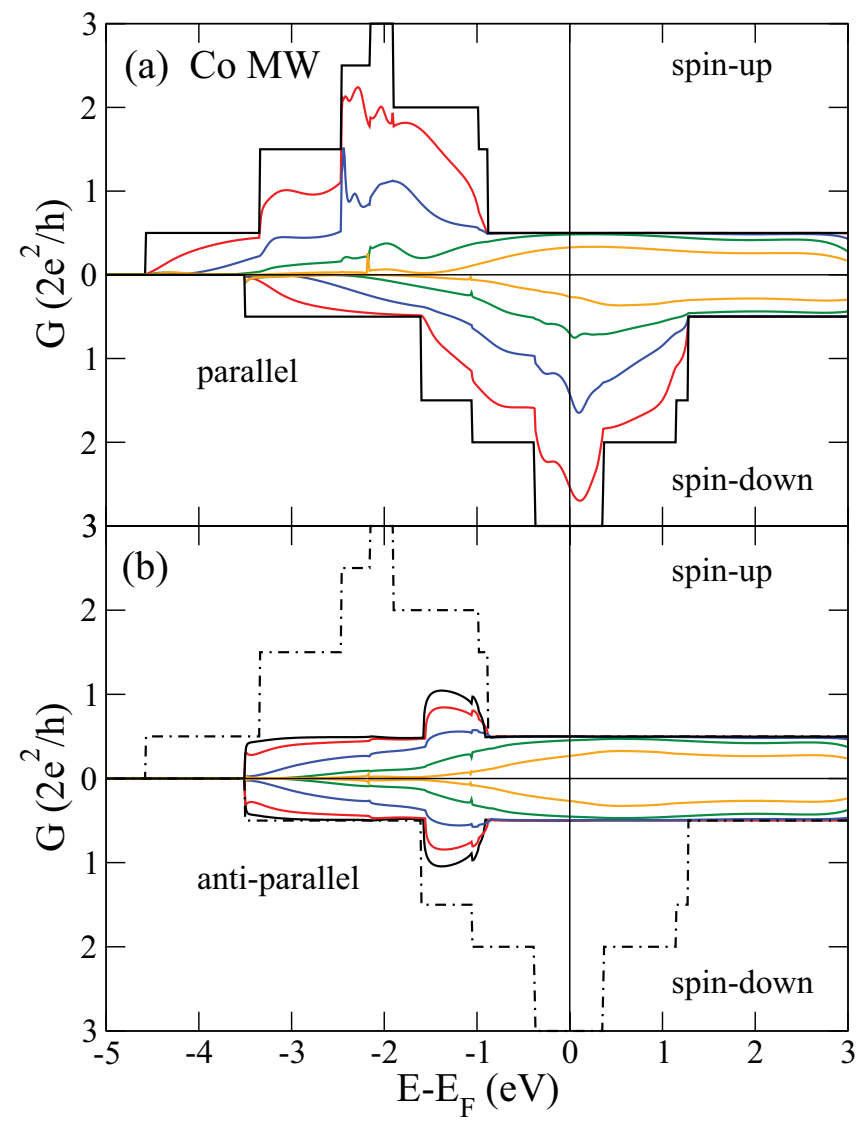

FIG. 11. (Color online) Conductance between two ferromagnetic Co monowires separated by a gap $\Delta$ in (a) parallel and in (b) antiparallel alignment of the magnetization. A supercell of 16 atoms has been used for the scattering region and the transport Hamiltonian was constructed based on FSWFs in the second-NN approximation. From left to right: gap of $\Delta=0.0,0.45,1.05,1.85$, and $2.85 \mathrm{bohr}$. Upper and lower part of the plots show the spin-up and spin-down transmission channels, respectively.

alignment due to the overlap between minority bands of $\Delta_{3}$ and $\Delta_{4}$ symmetry. This notion is confirmed by the calculated conductance in the two magnetic configurations as a function of gap size as shown in Fig. 11. At the Fermi level, we observe majority and minority spin conductances of $G_{\text {maj }}=e^{2} / h$ and $G_{\min }=6 e^{2} / h$, respectively, for a perfect ferromagnetic Co monowire [see Fig. 11(a)]. As the central bond is stretched, the minority conductance drops rapidly because it originates from the more localized $d_{x z, y z}$ and $d_{x y, x^{2}-y^{2}}$ states. The majority conductance, on the other hand, is due to $s-d_{z^{2}}$ states and decays much more slowly.

In the antiparallel alignment [Fig. 11(b)], the conductance is the same in both spin channels. There is only a small energy window between 1 and $1.7 \mathrm{eV}$ below the Fermi energy in which the $d_{x z, y z}$ and $d_{x y, x^{2}-y^{2}}$ states overlap, and at the Fermi energy, the conductance is dominated by the $s-d_{z^{2}}$ states. The conductance in the antiparallel alignment can be interpreted as an envelope of spin-up and spin-down transmission functions calculated for the parallel case as an electron can only be transmitted if there are states of the same symmetry in both spin channels. The conductance in this configuration is also in 


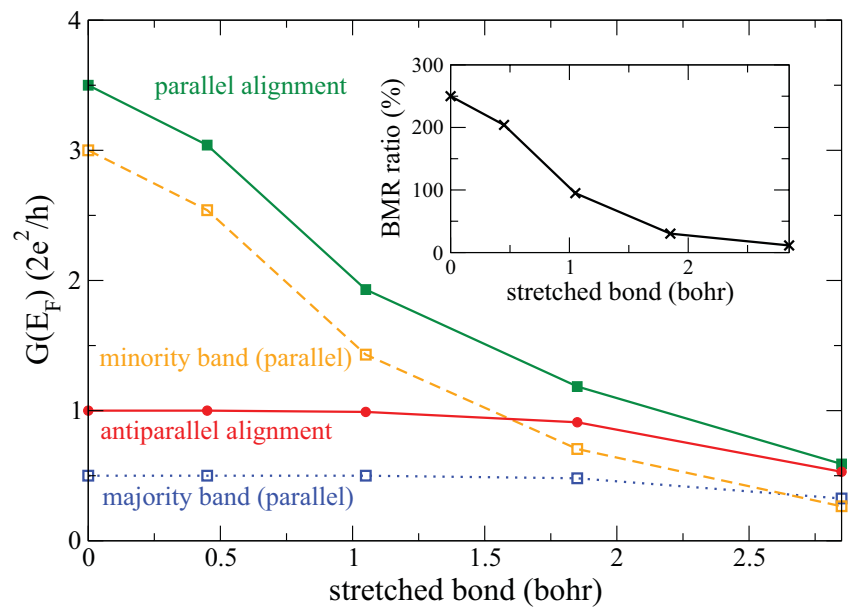

FIG. 12. (Color online) Conductance at $E_{F}$ for the antiparallel (red filled circles, solid line) and the parallel (green solid squares, solid line) alignments of two ferromagnetic Co monowires as a function of separation. For the parallel case, the decomposition into majority (blue open squares, dashed line) and minority (orange open squares, dotted line) contributions is given. The inset shows the BMR ratio as a function of separation.

good agreement with that reported by Smogunov et al. using a scattering approach and pseudopotentials. ${ }^{46}$

Based on the obtained quantum conductance at the Fermi level, we can calculate the ballistic magnetoresistance (BMR) upon stretching the central bond. The BMR is defined as the difference between the conductance in the parallel and antiparallel alignment divided by the antiparallel conductance:

$$
\mathrm{BMR}=\frac{G_{\mathrm{P}}\left(E_{F}\right)-G_{\mathrm{AP}}\left(E_{F}\right)}{G_{\mathrm{AP}}\left(E_{F}\right)} \times 100 \% .
$$

Figure 12 displays the evolution of the spin-resolved conductance as a function of gap size for the two magnetic configurations. As noted above, the parallel alignment is characterized by a rapidly decreasing minority spin conductance and a nearly constant majority spin contribution. However, the minority spin conductance dominates until the end of the bond length range which we considered. In the antiparallel alignment, the conductance of both spin channels is the same and behaves similar to the majority spin channel of the parallel alignment as it is due to $s-d_{z^{2}}$ states. From this analysis of the channel contribution, we can understand the fast drop of the BMR found upon stretching (inset of Fig. 12) of the central bond in the monowire.

\section{SPIN-ORBIT SCATTERING AT IMPURITIES}

In the previous sections, we applied our quantum transport code to systems with strong spin-orbit coupling (Pt monowires) and high spin polarization (Co monowires). In the following, we combine the two effects in order to study the scattering at impurities in the presence of spin-orbit coupling. We consider two types of model systems. We begin with nonmagnetic Pt monowire with a single Co impurity atom and calculate the dependence of the conductance on the magnetization direction of the Co atom. An analysis of the orbital decomposed transmission function allows us to study the influence of SOC on the different channels. We find that band mixing due to SOC has a pronounced influence, in particular, on the contribution from the band with $\Delta_{4}$ symmetry. As a second system, we consider a ferromagnetic Co monowire with a single Pt impurity atom and compute the conductance for the two magnetization directions of the Co wire, either along the direction of the wire or perpendicular to it. From our calculations of the conductance including spin-orbit coupling, we can also determine the ballistic anisotropic magnetoresistance (BAMR), i.e., the difference of transmission between a magnetization parallel to the current and perpendicular to the current.

While our systems are idealized, they can be seen as prototypical for experiments that may be performed for example by scanning tunneling microscopy in the contact regime ${ }^{7}$ or in break junctions. ${ }^{1,10,71}$ Scalar-relativistic calculations, i.e., neglecting SOC, in a similar geometry for a Ni impurity in a Au monowire have been performed before. ${ }^{73}$

\section{A. Magnetic impurity in a nonmagnetic wire}

We begin our investigation of spin-orbit scattering at an impurity by considering a single Co atom in a Pt monowire. This is the simpler of the two systems due to the nonmagnetic $\mathrm{Pt}$ leads. We have already discussed the conductance of $\mathrm{Pt}$ monowires with and without spin-orbit coupling in Sec. III. Here, we study the conductance for different magnetization directions of the Co impurity atom in order to calculate the socalled ballistic anisotropic magnetoresistance, which has been predicted based on DFT calculations ${ }^{9}$ and was experimentally reported for Co break junctions. ${ }^{10}$

Before we discuss the calculated conductance, we focus on the magnetic properties of our system. From the DFT calculations in the scalar-relativistic case, we obtain spin moments of $2.46 \mu_{B}$ for the Co atom which induces Pt spin moment of a magnitude of up to $0.27 \mu_{B}$, oscillating in sign as a function of separation from the Co atom. A similar behavior was found upon including SOC in the calculations for both magnetization directions, with a Co spin moment of about $2.49 \mu_{B}$. Including spin-orbit interaction in the calculations gives rise to finite values of the orbital moments of the atoms, which play an important role in determining the energetically favorable direction of the magnetization. ${ }^{70}$ In our system, the orbital moments of the $\mathrm{Co}$ atoms are much larger than those of the surrounding Pt atoms, and constitute $0.12 \mu_{B}$ and $0.19 \mu_{B}$ for the magnetization along the chain axis $(z)$ and perpendicular to it $(r)$, respectively. Accordingly, ${ }^{70}$ this results in an energetical preference of the in-chain magnetization direction over the out-of-chain direction, with a calculated magnetocrystalline anisotropy energy (MAE) of $4.3 \mathrm{meV}$ per magnetic atom.

We now turn to the calculated conductance presented in the three top panels of Fig. 13 for the scalar-relativistic case and upon including spin-orbit coupling for the two different magnetization directions. For reference, the orbitally decomposed conductance and the density of states (DOS) of a perfect $\mathrm{Pt}$ monowire is given in each of the three plots and in the panels below, respectively. As a general trend, the introduction of a Co scatterer results in a nonperfect matching between the spin-split Co $3 d$ states and the more delocalized Pt $5 d$ states 


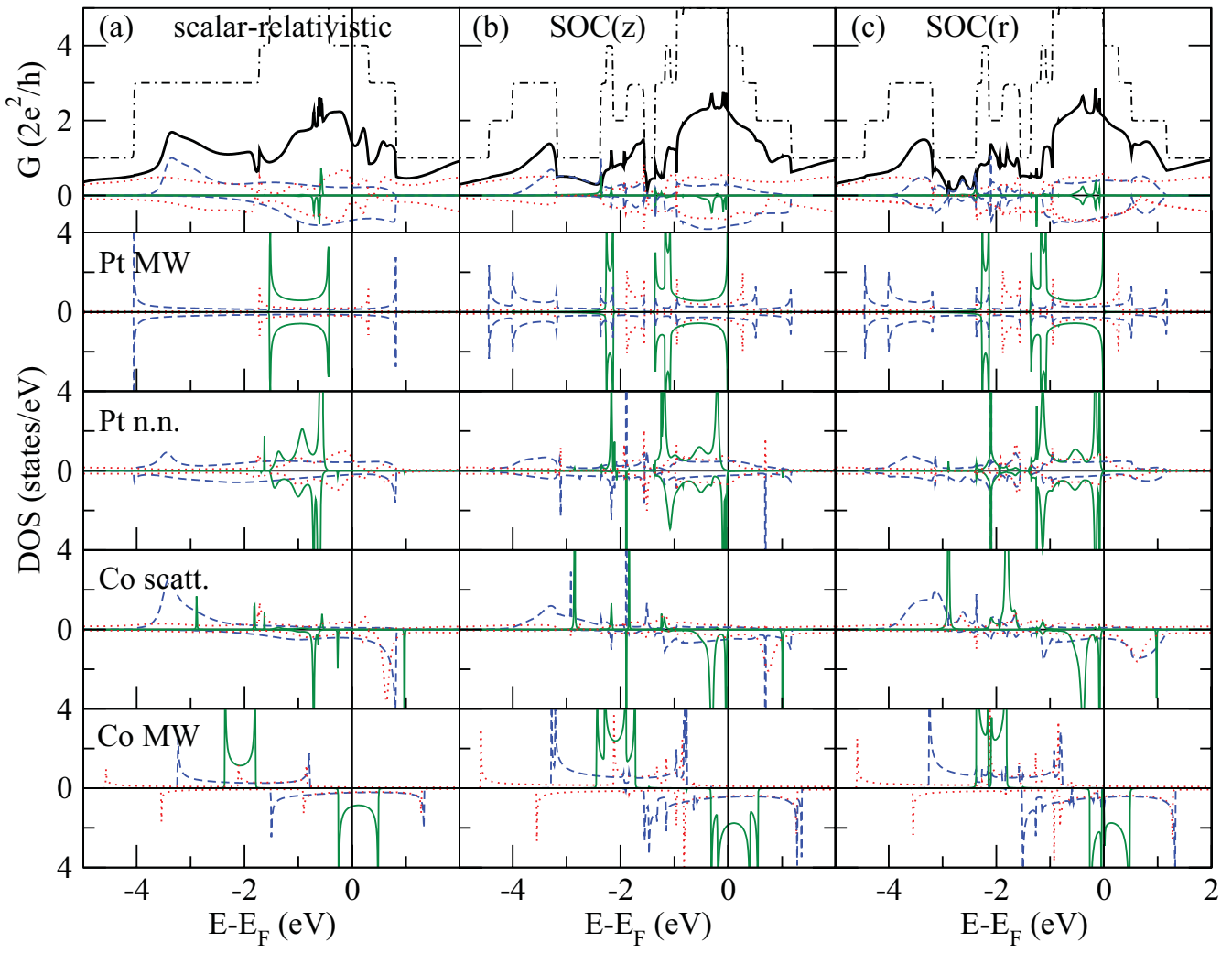

FIG. 13. (Color online) Conductance of a Pt monowire with a single Co impurity in (a) the scalar-relativistic (SR) approximation and including spin-orbit coupling (SOC) for a magnetization (b) along the chain axis $(z)$ and (c) perpendicular to it $(r)$. In addition to the total conductance (black thick line), each panel shows the transmission for a perfect Pt monowire (dashed-dotted line) and orbital decomposed into the $\Delta_{1}$ (red dotted line), $\Delta_{3}$ (blue dashed line), and $\Delta_{4}$ band (green solid line) contribution. The projection onto the spin-up and spin-down states is given for two different directions of the $y$ axis, respectively. Below each conductance panel, the density of states (DOS) is displayed in the corresponding electronic configuration, i.e., SR or SOC, for a perfect Pt monowire, the Pt atom adjacent to the Co impurity, the Co impurity, and a perfect Co monowire. The DOS is orbital decomposed similarly to the transmission.

(cf. the band structures in Figs. 3 and 10). In all three cases, a clear signature of the exchange-split Co $\Delta_{3}$ band can be observed in the overall conductance, most clearly visible in the spin and orbital decomposition. As expected, the $\Delta_{4}$ bands are shifted toward the Fermi energy upon including spin-orbit coupling, but due to the energetical mismatch between the Co and $\mathrm{Pt} \Delta_{4}$ bands in SR and for both magnetization directions with SOC, this band plays only a minor role in the overall conductance.

Nevertheless, there is a considerable difference between the conductance at the Fermi level in the scalar-relativistic case $G_{\mathrm{SR}}=1.40 G_{0}$, and upon including SOC either for $z$ magnetization $G_{\|}=2.25 G_{0}$, or $r$ magnetization $G_{\perp}=2.10 G_{0}$, as seen in Fig. 14. The main reason for this large difference between SR and SOC conductances can be found in the $\Delta_{1}$ band of SR Pt. In this channel, the DOS is reduced compared to the SOC cases at the Fermi energy at the Pt nn atoms and there is a corresponding reduction of the conductance, as shown in Fig. 13. The difference of $G_{\|}-G_{\perp}=0.15 G_{0}$ between the two different magnetization directions can be found in the larger minority $\Delta_{3}$-state contribution of the parallel aligned axis. Here, the SR and the parallel SOC case behave similarly. The DOS for $\Delta_{3}$ majority states is small at the Fermi level,

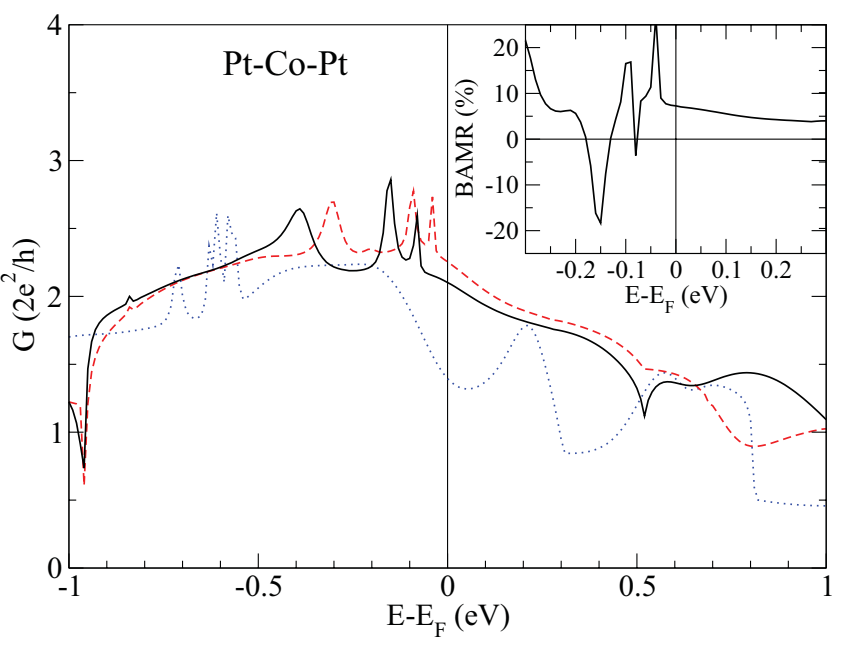

FIG. 14. (Color online) Conductance around the Fermi level for a Pt wire with a single Co impurity atom without spin-orbit coupling (dotted blue line) and including SOC for an in-chain (dashed red line) and an out-of-chain (solid black line) magnetization direction. The inset shows the ballistic anisotropic magnetoresistance (BAMR) as defined by Eq. (24). 
the majority state conductance is reduced in comparison to the minority state contribution, as a result of the exchange splitting of the Co scatterer.

Interestingly, this is not the case for the $r$ direction of the magnetization for which majority and minority channels contribute equally to the total conductance. This effect also occurs for the $\Delta_{3}$ minority channel between -2.8 and $-3.9 \mathrm{eV}$ as well as for the $\Delta_{4}$ conductance just below the Fermi energy. While the very sharp spin-up $\Delta_{4}$ peak in the SR transmission at $-0.7 \mathrm{eV}$ can be traced back to a small spin-up $\Delta_{4}$ peak in the DOS of the central Co atom at this energy, this is not the case for the mentioned regions in case of the $r$ magnetization, for which no majority $\Delta_{3}$ and $\Delta_{4}$ states are present at the scatterer. The origin of this effect is the broken cylindrical symmetry when the magnetization points out of chain. This broken symmetry allows for a hybridization between $\Delta_{1}$ and $\Delta_{3}$ bands with $j=\frac{1}{2}$, as well as between $\Delta_{3}$ and $\Delta_{4}$ bands with $j=\frac{3}{2}$. As a result, an incident electron of $j=\frac{1}{2}\left(\frac{3}{2}\right)$ can be transmitted into a state with $j=\frac{1}{2}\left(\frac{3}{2}\right)$ of different orbital character and spin. This channel for scattering is less effective than the spin-conserving scattering for the in-chain magnetization, resulting in a larger conductance in this case.

The changes in the ballistic conductance due to ballistic spin scattering are important for the ballistic anisotropic magnetoresistance. The BAMR is defined analogously to the anisotropic magnetoresistance as

$$
\mathrm{BAMR}=\frac{G_{\|}-G_{\perp}}{G_{\perp}} \times 100 \%,
$$

where $G_{\|}$and $G_{\perp}$ are the conductances for the magnetization along the wire axis and perpendicular to it, respectively. ${ }^{9}$ The difference of $0.15 G_{0}$ at the Fermi level in favor of the parallel quantization axis due to ballistic spin scattering results in a small BAMR of the order of $7 \%$ (see inset of Fig. 14). A small shift between the $\Delta_{4}$ contributions due to a small spin splitting of those bands for the Pt atom neighboring the Co scatterer (cf. Fig. 13) results in an oscillatory behavior of the BAMR when the energy is varied from -0.05 to $-0.2 \mathrm{eV}$, with BAMR ranging from $-20 \%$ to $25 \%$.

\section{B. Nonmagnetic impurity in ferromagnetic wire}

In the previous example, we have seen that the transmission can be affected by ballistic spin scattering, leading to a small BAMR below the Fermi energy and BAMR oscillations due to a shift in the $\Delta_{4}$ orbitals of the Pt atom next to the Co scatterer. In this section, we consider a nonmagnetic scatterer, a Pt atom, in a ferromagnetic Co monowire. We find that this situation leads to an enhanced BAMR close to the Fermi level, which is crossed by the $\Delta_{4}$ band. In this case, we do not expect strong ballistic spin scattering because of the magnetic leads since large exchange splitting prohibits scattering between the states with opposite spin.

First, we consider the junction in the scalar-relativistic approximation in order to understand the main impact of the Pt scatterer on the conductance. While Co atoms in the leads carry a magnetic moment of $2.13 \mu_{B}$, the Co atoms in the vicinity of the $\mathrm{Pt}$ atom have moments in the range of 2.15-2.20 $\mu_{B}$, and the Pt atom itself is spin polarized with a considerable moment of $0.36 \mu_{B}$. As can be seen in the orbitally decomposed conductance [Figs. 15(a)-15(c)], the reduction of the transmission due to the $\mathrm{Pt}$ impurity atom is relatively small compared to the perfect ferromagnetic Co monowire. We can understand this general behavior from the fact that the Pt $5 d$ bands possess a broader bandwidth and thereby allow transmission in the entire regime of the spin-polarized Co $3 d$ bands [cf. Figs. 3 and 10).

In the $s-d_{z^{2}}$ channel, the reduction of the transmission is similarly small for the majority and minority spin contributions due to the energetic alignment of the spin-split states of the Co wire with the states of the Pt impurity. In the majority spin channel, a significant reduction of transmission only occurs in a region from $E_{F}-2.1 \mathrm{eV}$ to $E_{F}-0.9 \mathrm{eV}$ where the perfect conductance amounts to $G_{0}$. In the spin- and orbitaldecomposed density of states [Figs. $15(\mathrm{~d})$ and $15(\mathrm{~g})$ ], we also find two resonances at the Pt impurity located at 2.8 and $2.3 \mathrm{eV}$ below the Fermi energy in the majority and minority spin channels, respectively. In the conductance, we observe a Fanotype line shape due to the coupling of the $\Delta_{1}$ band to these resonances.

The conductance from the $\Delta_{3}$ bands displays only a reduction at the bottom and top of the band in both spin channels as the onsite energies of Co and Pt $d_{x z, y z}$ states are close in energy. The density of states of the Pt atom [Fig. 15(h)] shows that the $d_{x z, y z}$ states are spin split, carry a significant part of the Pt moment, and align well with the $\Delta_{3}$ bands in the Co monowire, resulting in an efficient transport channel. The most severe change in the conductance upon introducing a Pt impurity occurs in the $\Delta_{4}$ band. Here, we observe a large decrease due to scattering at the Pt impurity. For both the $d_{x z, y z}$ and $d_{x y, x^{2}-y^{2}}$ channels, bound states on the Pt atom can be found due to the lower onsite potential at the Pt site. For the $\Delta_{3}$ symmetry, there are such states at $-4.1 \mathrm{eV}$ for the majority band and at $-3.3 \mathrm{eV}$ for both spin channels, which do not contribute to the conduction as they are below the $\Delta_{3}$ band of the Co leads. For the $\Delta_{4}$ symmetry, there are majority states around $-2.5 \mathrm{eV}$ and a paired state at $-2 \mathrm{eV}$ with respect to the Fermi level, not contributing to the majority channel transmission.

$\Delta_{4}$ electrons are only transmitted in the small overlap region around $-2.1 \mathrm{eV}$ for majority and around Fermi level for minority states, where a very narrow band is formed in both cases. The shape of the transmission function follows the two-peak (majority band) and three-peak (minority band) shapes of the DOS of the central Pt atom.

Now, we turn to the effect of spin-orbit coupling on the magnetic and transport properties of the Co-Pt-Co junction. For the perfect Co monowire, we found a magnetocrystalline anisotropy energy, i.e., the difference in energy for the magnetization in the chain axis and perpendicular to it, of $0.8 \mathrm{meV}$ per magnetic atom in favor of an out-of-chain magnetization and orbital moments of $0.17 \mu_{B}$ for the out-of-chain and $0.22 \mu_{B}$ for in-chain direction. Upon introducing the Pt atom, this value is reduced to $0.5 \mathrm{meV}$ per magnetic atom, which is consistent with our observation in the previous section for a $\mathrm{Pt}-\mathrm{Co}-\mathrm{Pt}$ junction favoring the in-chain direction. The magnetic moment of the $\mathrm{Pt}$ atom is $0.36 \mu_{B}$ for both magnetization directions, and we find similar orbital moments of $0.09 \mu_{B}$ (out of chain) and $0.10 \mu_{B}$ (in chain). Characteristically, as in the case of the Co leads, the orbital moments of the Co atoms adjacent to the 


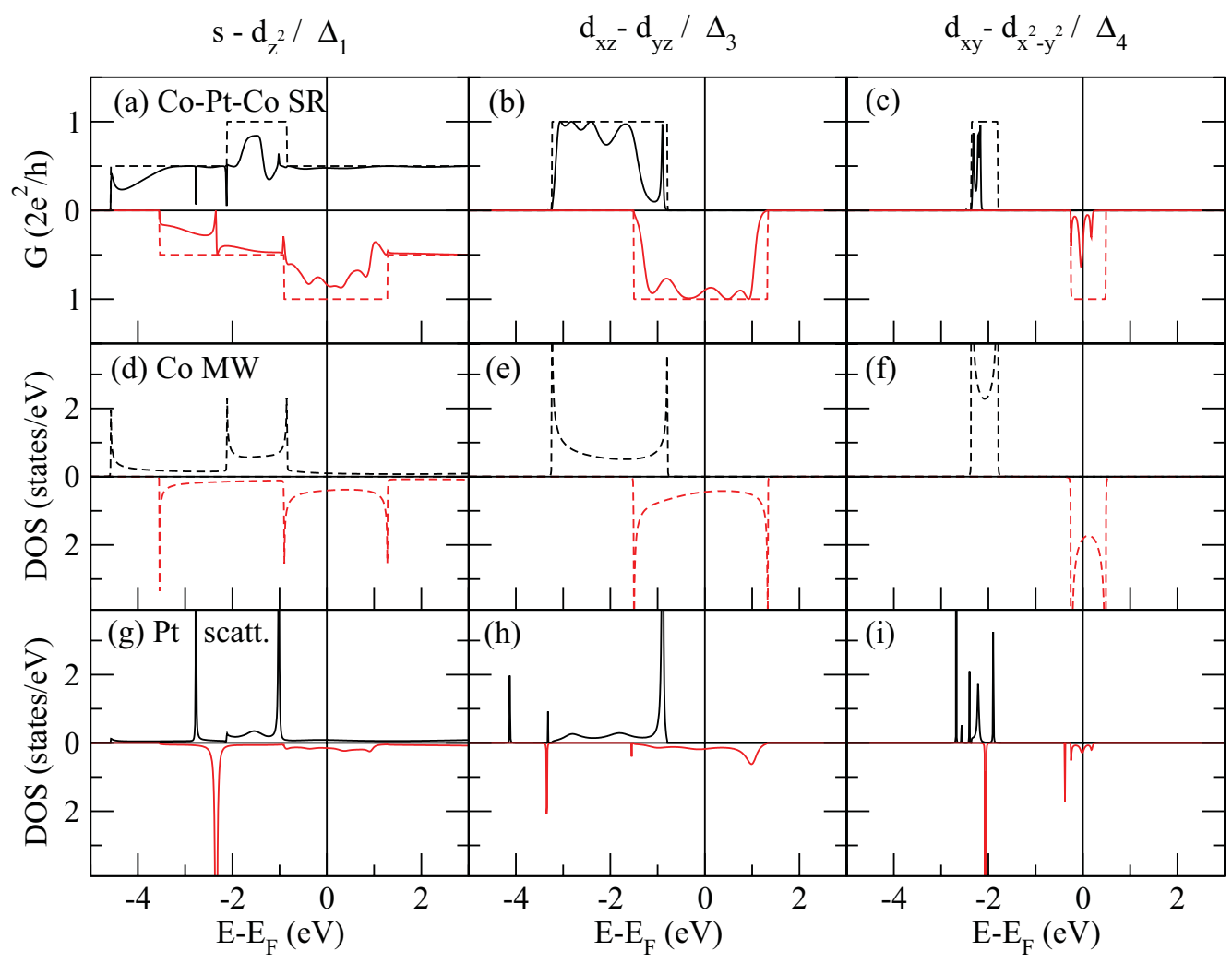

FIG. 15. (Color online) (a)-(c) Orbital decomposition of transmission through a ferromagnetic Co wire with a single Pt impurity (solid lines) and a perfect Co wire (dashed lines) for the $\Delta_{1}, \Delta_{3}$, and $\Delta_{4}$ channels, respectively, for spin up (black, upper part) and spin down (red, lower part). (d)-(f) Density of states of the Co leads orbitally decomposed for spin-up (dashed black lines, upper part) and spin-down (red dashed lines, lower part) states. (g)-(i) Orbitally decomposed DOS of the Pt impurity atom, for spin up (black, upper part) and spin down (red, lower part).

Pt impurity are significantly larger for the in-chain direction (reaching as much as $0.53 \mu_{B}$ for the nearest Co atom) than for the out-of-chain direction (at most $0.2 \mu_{B}$ ). This means that the out-of-chain easy magnetization axis in our scattering region is mainly due to the Co atoms.

For the transport properties including SOC, the bands with $\Delta_{3}$ and $\Delta_{4}$ symmetry are essential. Depending on the quantization axis defined by the magnetization direction, the degeneracy of these bands is lifted. In contrast to the Pt-Co-Pt system, the Co electrodes are ferromagnetic and therefore the splitting in the steplike conductance in the perfect Co wires changes upon switching the quantization axis from along the chain axis to perpendicular to it.

As can be seen from Fig. 16, changing the magnetization direction in a perfect infinite Co chain leads to a reduction of the transmission from $3.5 G_{0}$ (along the chain) to $1.5 G_{0}$ (perpendicular to the chain) in a very small energy window around the Fermi energy, which results in a huge value of the ballistic anisotropic magnetoresistance of $133 \% .{ }^{10}$ In a realistic situation, however, such values of the anisotropic magnetoresistance can be hardly achieved, owing to the destruction of perfect conducting channels by imperfections, impurities, and disorder.

In the case of a Co chain with a Pt impurity, similarly to the scalar-relativistic case, we observe a reduction by roughly a factor of 2 in the overall conductance over the entire energy range due to the less efficient coupling between the Co wire and the Pt impurity compared to an perfect Co wire, especially for the $\Delta_{3}$ and $\Delta_{4}$ orbitals. At the Fermi energy, we find majority and minority spin contributions from the $\Delta_{1}$ band of about 0.5 and $1.0 G_{0}$ for both magnetic directions. Only the minority states of the other two orbital symmetries are present due to the exchange splitting. The minority $\Delta_{3}$ band contributes almost $1.0 G_{0}$ for the in-chain magnetization, while it reveals a large dip at $E_{F}$ for the out-of-chain magnetization. Accordingly, the $\Delta_{4}$-band conductance also changes significantly upon switching the magnetization direction, owing to the changes in the details of hybridization between $\Delta_{3}$ and $\Delta_{4}$ states when the direction of the magnetization is changed [see Figs. 16(b) and 13 (cf. DOS of the Co monowires for the two different magnetization directions)]. These changes in the energetic structure of $\Delta_{3}$ and $\Delta_{4}$ states lead to a large difference between the in-chain and out-of-chain conductances, also visible for the pure Co chain in the Fig. 16.

In Fig. 17, the conductance is displayed in a small energy window around the Fermi energy for the two different magnetization directions. It is apparent that the changes arise due to the modifications of the $\Delta_{4}$-band conductance between Fermi level and $-0.05 \mathrm{eV}$, and $\Delta_{3}$-band conductance around $E_{F}$ and $-0.15 \mathrm{eV}$, which are subject to different band mixing from spin-orbit coupling. As a result of the fine structure of the $\Delta_{4}$ and $\Delta_{3}$ conductances [see Fig. 16(b)], the BAMR which 


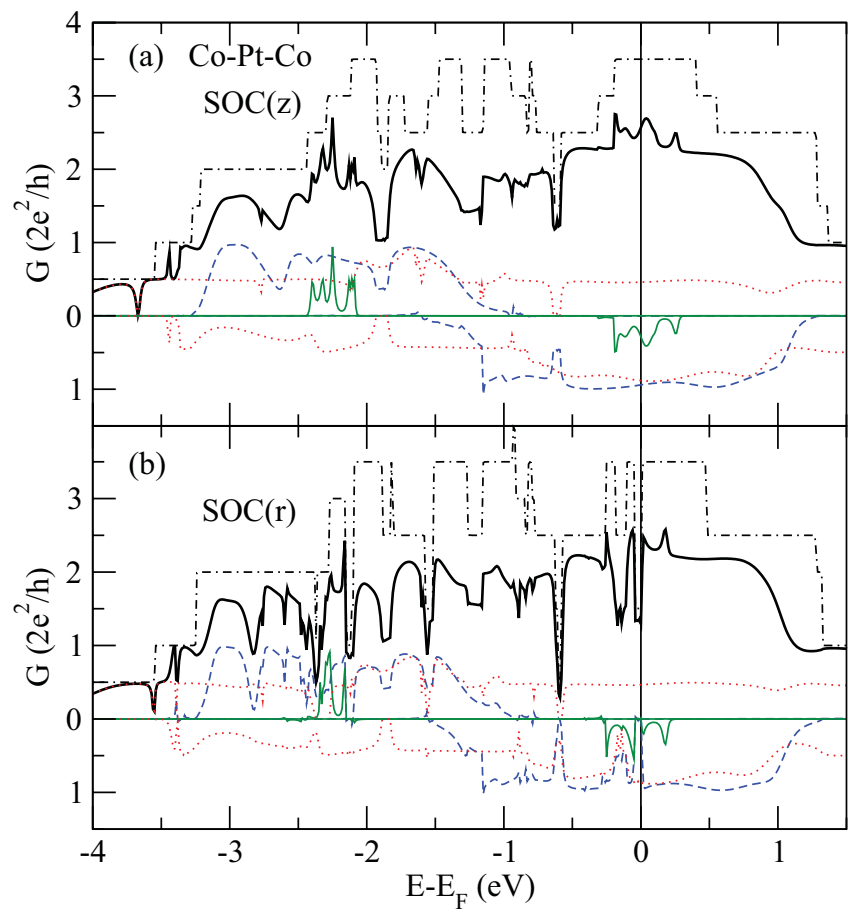

FIG. 16. (Color online) Conductance including SOC for a Co monowire with a Pt impurity atom for (a) magnetization along the wire axis and (b) perpendicular to the axis. The decomposition of transmission into $\Delta_{1}$ (dotted red lines), $\Delta_{3}$ (dashed blue lines), and $\Delta_{4}$ (solid green lines) channels for majority spin (positive $y$ axis) and minority spin (negative $y$ axis) shows the presence of a $\Delta_{4}$ minority band channel at the Fermi level. Black dashed-dotted lines display the transmission of the perfect infinite Co leads.

we obtain, shown in the inset of Fig. 17, displays a strong variation with energy. Compared to the BAMR of a perfect Co MW of $133 \%$, a Pt scatterer reduces this effect to $80 \%-100 \%$,

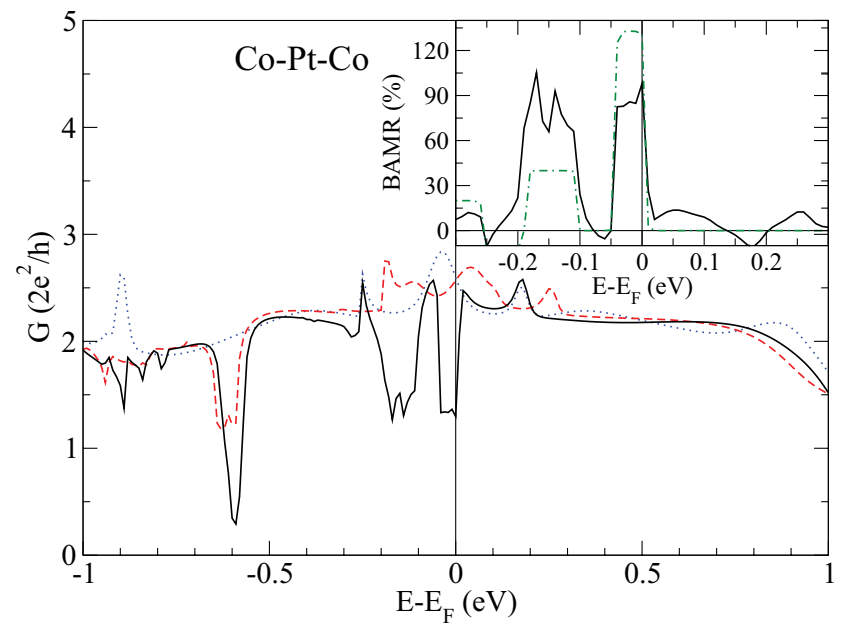

FIG. 17. (Color online) Conductance around the Fermi level for a Co monowire with a Pt impurity atom in the scalar-relativistic approximation (blue dotted line) and including SOC for a magnetization along the chain axis (dashed red line) and perpendicular to the axis of the wire (solid black line). The inset shows the BAMR as defined by Eq. 17 for the Co monowire with a Pt impurity (solid black line) and for a perfect infinite Co monowire (dashed-dotted green line). which is still considerably high. An enhanced BAMR can be found for the second peak below the Fermi energy, where a $\Delta_{4}$-conduction peak for the in-chain direction in coincidence with a $\Delta_{3}$-conduction depletion result in a BAMR increase from $40 \%$ for the perfect Co MW to $60 \%-100 \%$ when a Pt scatterer is introduced.

\section{SUMMARY}

We have implemented the Landauer-Büttiker method to calculate the ballistic electron transport through one-dimensional nanoscale junctions based on density functional theory calculations within the full-potential linearized augmented (FLAPW) method. In order to apply the efficient Green's function method to calculate the conductance, we have mapped the extended Bloch states obtained from the FLAPW method to the minimal basis set of localized Wannier functions and constructed the Hamiltonian for the open system. With our approach, it is feasible to calculate ballistic transport through one-dimensional nanoscale systems including magnetism and spin-orbit coupling with the accuracy and flexibility of the FLAPW method.

We apply our method to calculate the conductance of nonmagnetic Pt monowires with a single stretched bond, including spin-orbit coupling. Already, this simple example shows the key impact of SOC for systems containing heavy transition metals. As a second example, we considered a Co monowire and studied the magnetoresistance upon stretching the wire at a single bond. The decomposition of the transmission into the channels of different orbital symmetry shows the dominant contribution of $s$ and $d_{z^{2}}$ states as one moves from the contact to the tunnel regime. Finally, we studied the effect of spin-orbit scattering at an impurity atom in a monowire. We considered two model cases: (i) a magnetic atom in a nonmagnetic wire, Co in a Pt monowire, and (ii) a nonmagnetic heavy element in a ferromagnetic wire, $\mathrm{Pt}$ in a Co monowire. We observed for both cases a distinct dependence of the conductance on the magnetization direction with respect to the wire axis.

We found for a Co impurity in a Pt chain that due to the broken cylindrical symmetry for an out-of-chain magnetization direction, the hybridization between states of different angular character and spin but with identical quantum number $j$ leads to scattering processes that do not conserve spin. Those ballistic spin-scattering processes are resulting into a BAMR of $7 \%$. The relatively moderate values are caused by the large background conductance from bands originating from $s-d_{z^{2}}$ and $d_{x z, y z}$ states, which are not modified much upon switching the magnetization. On the other hand, for a Pt impurity in a Co chain, we find that the presence of an impurity, although reducing somewhat the BAMR of the pure Co chain, still leads to values of BAMR of about $100 \%$, which originates from hybridization between the $\Delta_{3}$ and $\Delta_{4}$ states moderated via SOC by the direction of the magnetization.

\section{ACKNOWLEDGMENTS}

We acknowledge helpful discussions with S. Blügel. Funding by the DFG within the SFB677 is gratefully acknowledged. S.H. thanks the DFG for financial support under HE3292/8-1. N.-P.W. is grateful for financial support from The Natural 
Science Foundation of Zhejiang Province in China under Grant No. Y6100467. Y.M. and F.F. gratefully acknowledge the Jülich Supercomputing Centre for computing time and funding under the HGF-YIG Programme VH-NG-513.

\section{APPENDIX: COMPUTATIONAL DETAILS}

\section{Pt monowires}

Nonmagnetic (NM) 6- and 12-atom-supercell calculations with an interatomic distance of $d_{\mathrm{Pt}}=4.48 \mathrm{bohr}$ and the central bond stretched by $\Delta=0.0,0.34,0.72,1.22,1.82$, and 2.52 bohr. We applied the generalized gradient approximation (GGA) to the exchange-correlation potential. ${ }^{74}$ For calculations in the scalar-relativistic (SR) approximation, the irreducible part of the 1D Brillouin zone (BZ) was sampled by $6-10 k$ points depending on the size of the supercell. For the 6-atom supercell, we also performed calculations including spin-orbit coupling in second variation. For calculations with SOC, the whole 1D BZ was sampled by $24 k$ points. In all calculations, $G_{\max }$ was chosen to be $3.7 \mathrm{bohr}^{-1}$, which corresponds to approximately 200 basis functions per atom. The diameter of the cylindrical vacuum $D_{\text {vac }}$, and the value of the in-plane auxiliary lattice constant $\tilde{D},{ }^{54}$ were set to 5.0 and 7.3 bohr, respectively.

For the conductance calculations, we applied the locking technique to a perfect monowire to describe the semi-infinite leads (see Sec. IID). In the SR approximation, FSWFs and MLWFs were generated on a mesh of $16 k$ points in the whole BZ starting from one $4 s$ and $53 d$ orbitals per atom in the supercell, based on solutions of the radial equation of the first-principles potential as trial functions. In the calculations including SOC, MLWFs were generated on a $24 k$-point mesh in the whole BZ based on 2 radial $4 s$ and 10 radial $3 d$ orbitals per atom, based on solutions of the radial equation of the first-principles potential as trial functions, due to the coupled spin channels. The energy bands were disentangled using the procedure described in Ref. 65. For the SR calculations, the lowest 80 eigenstates are needed for $72 \mathrm{WFs}$ for the 12 -atom supercell and the lowest 44 eigenvalues per $k$ point for $36 \mathrm{WFs}$ for the 6-atom-supercell calculations. With SOC, the lowest 80 eigenstates per $k$ point for 72 WFs were used.

\section{Co monowires}

Calculations with a lattice constant of $d_{\mathrm{Co}}=4.15 \mathrm{bohr}$ and a central stretched bond with stretching $\Delta=0.0,0.45,1.05$, 1.85 , and 2.85 bohr. Two collinear magnetic configurations of the Co monowire are considered, parallel or antiparallel alignment of the Co spins on the left and on the right sides of the gap, described by performing two calculations: An 8-atom supercell constructed from two 4-spin blocks separated by a gap and aligned in parallel (up), while in order to mimic the antiparallel alignment, we considered 16 atoms in the supercell with 4-spin (up), 8-spin (down), and 4-spin (up) blocks, separated by two gaps with the spins antiparallel to each other at each side of the gap.

The perfect lead ferromagnetic Co monowire was calculated with $24 k$ points in the whole BZ, using the $G_{\max }$ of $4.1 \mathrm{bohr}^{-1}(\approx 220$ basis functions per atom). For both 8and 16-atom-supercell calculations, the irreducible part of the $1 \mathrm{D}$ Brillouin zone was sampled by $8 k$ points and $G_{\max }$ was chosen to be 3.7 bohr $^{-1}$, resulting in approximately 210 basis functions per atom. The vacuum parameters $D_{\text {vac }}$ and $\tilde{D}$ constituted 4.3 and 6.6 bohr, respectively, in all cases. The exchange-correlation potential was treated within the GGA. ${ }^{74}$ For all quantum conductance calculations, the locking technique (see Sec. IID) to a perfect FM Co monowire was used. As trial orbitals for the FSWFs, $6 s$ and $d$ orbitals per atom and spin in the supercell were used, based on solutions of the radial equation of the first-principles potential. For the disentanglement procedure, ${ }^{65}$ the lowest 58 (110) eigenstates per $k$ point were used to obtain the 48 (96) WFs in the 8- (16-) atom-supercell calculation.

\section{Scattering on impurities}

A 9-atom supercell was used for the scattering region consisting of one impurity atom ( $\mathrm{Pt}$ or $\mathrm{Co}$ ) and four monowire atoms $(\mathrm{Co}$ or $\mathrm{Pt})$ on both sides. The interatomic distance was chosen as $d_{\mathrm{Co}}=4.15$ bohr for the Co monowire with a $\mathrm{Pt}$ impurity and as $d_{\mathrm{Pt}}=4.48$ bohr for the Pt monowire with a Co impurity. The exchange-correlation potential was treated within the GGA, ${ }^{74}$ and SOC was included in second variation. All calculations were performed in the scalar-relativistic approximation and for two different directions of the magnetization with SOC, along the chain axis and perpendicular to it. The 1D Brillouin zone was sampled by $16 k$ points and $G_{\max }$ was set to $3.9 \mathrm{bohr}^{-1}$, resulting in approximately 175 (190) basis functions per atom for the $\mathrm{Co}(\mathrm{Pt})$ monowire with a Pt (Co) impurity. For the case of an isolated Pt impurity, the leads were described by a Co monowire in a 3-atom unit cell in either the SR approximation or including SOC for the magnetization direction along the wire axis or perpendicular to it. The BZ was sampled by $24 k$ points and $G_{\max }$ was set to $4.1 \mathrm{bohr}^{-1}$, resulting in approximately 210 basis functions per atom. For Pt monowire with a Co impurity, the lead's electronic structure was obtained from calculations of perfect Pt monowires. The vacuum parameters for all cases constituted 4.3 and 6.6 bohr for $D_{\text {vac }}$ and $\tilde{D}$, respectively.

For all quantum conductance calculations, the locking technique was used and the third-nearest-neighbor approximation was employed. In the SR case, FSWFs were generated on a $16 k$-point mesh in the whole 1D BZ with one $s$ and five $d$ orbitals per atom and spin, based on solutions of the radial equation of the first-principles potential. For the disentanglement procedure, ${ }^{65}$ the lowest 64 (62) eigenvalues per $k$ point for 54 (54) WFs for $\mathrm{Pt}(\mathrm{Co})$ impurities in $\mathrm{Co}(\mathrm{Pt})$ monowires were considered. The Pt and Co lead WFs were constructed as described in Secs. 1 and 2 in this case. With SOC, the FSWFs were generated on a $16 k$-point mesh in the whole $1 \mathrm{D} \mathrm{BZ}$ with $2 s$ and $10 d$ orbitals per atom, based on solutions of the radial equation of the first-principles potential. For disentanglement, ${ }^{65}$ the lowest 116 eigenstates per $k$ point for 108 WFs were used. The WFs for the semi-infinite Co leads were generated on a $24 k$-point mesh with the same trial functions as those used for the atoms inside the scattering region, while for disentanglement, the lowest 26 eigenvalues per $k$ point for $18 \mathrm{WFs}$ per spin (SR) and the lowest 44 eigenvalues per $k$ point for 36 WFs (SOC) were used. The $\mathrm{Pt}$ lead WFs were constructed as described in Sec. I. 
*bhardrat@ theo-physik.uni-kiel.de

${ }^{1}$ T. Kizuka, Phys. Rev. B 77, 155401 (2008).

${ }^{2}$ N. Néel, J. Kröger, and R. Berndt, Phys. Rev. Lett. 102, 086805 (2009).

${ }^{3}$ J. Kröger, N. Néel, and L. Limot, J. Phys.: Condens. Matter 20, 223001 (2008).

${ }^{4}$ K. Tao, I. Rungger, S. Sanvito, and V. S. Stepanyuk, Phys. Rev. B 82, 085412 (2010).

${ }^{5}$ H. D. Chopra, M. R. Sullivan, J. N. Armstrong, and S. Z. Hua, Nat. Mater. 4, 832 (2005).

${ }^{6}$ M. Calvo, J. Fernández-Rossier, J. Palacios, D. Jacob, D. Natelson, and C. Untiedt, Nature (London) 458, 1150 (2009).

${ }^{7}$ M. Ziegler, N. Néel, C. Lazo, P. Ferriani, S. Heinze, J. Kröger, and R. Berndt, New J. Phys. 17, 085011 (2011).

${ }^{8}$ S. Schmaus, A. Bagrets, Y. Nahas, T. K. Yamada, A. Bork, M. Bowen, E. Beaurepaire, F. Evers, and W. Wulfhekel, Nat. Nanotechnol. 6, 185 (2011).

${ }^{9}$ J. Velev, R. F. Sabirianov, S. S. Jaswal, and E. Y. Tsymbal, Phys. Rev. Lett. 94, 127203 (2005).

${ }^{10}$ A. Sokolov, C. Zhang, E. Tsymbal, J. Redepenning, and B. Doudin, Nat. Nanotechnol. 2, 171 (2007).

${ }^{11}$ M. Büttiker, Y. Imry, R. Landauer, and S. Pinhas, Phys. Rev. B 31, 6207 (1985).

${ }^{12}$ Y. Meir and N. S. Wingreen, Phys. Rev. Lett. 68, 2512 (1992).

${ }^{13}$ P. Sautet and C. Joachim, Phys. Rev. B 38, 12238 (1988).

${ }^{14}$ E. G. Emberly and G. Kirczenow, Phys. Rev. B 58, 10911 (1998).

${ }^{15}$ K. Hirose and M. Tsukada, Phys. Rev. Lett. 73, 150 (1994).

${ }^{16}$ H. J. Choi and J. Ihm, Phys. Rev. B 59, 2267 (1999).

${ }^{17}$ N. Kobayashi, M. Brandbyge, and M. Tsukada, Phys. Rev. B 62, 8430 (2000).

${ }^{18}$ N. D. Lang, Phys. Rev. B 52, 5335 (1995).

${ }^{19}$ H. Dalgleish and G. Kirczenow, Phys. Rev. B 72, 155429 (2005).

${ }^{20}$ P. A. Khomyakov, G. Brocks, V. Karpan, M. Zwierzycki, and P. J. Kelly, Phys. Rev. B 72, 035450 (2005).

${ }^{21}$ L. V. Keldysh, Sov. Phys. JETP 20, 1018 (1965).

${ }^{22}$ L. Kadanoff and B. Baym, Quantum Statistical Mechanics (Benjamin, New York, 1962).

${ }^{23}$ T. Frederiksen, M. Brandbyge, N. Lorente, and A.-P. Jauho, Phys. Rev. Lett. 93, 256601 (2004).

${ }^{24}$ T. Frederiksen, M. Paulsson, M. Brandbyge, and A.-P. Jauho, Phys. Rev. B 75, 205413 (2007).

${ }^{25}$ K. S. Thygesen and A. Rubio, Phys. Rev. B 77, 115333 (2008).

${ }^{26}$ H. U. Baranger and A. D. Stone, Phys. Rev. B 40, 8169 (1989).

${ }^{27}$ F. Evers, F. Weigend, and M. Koentopp, Phys. Rev. B 69, 235411 (2004).

${ }^{28}$ A. Bagrets, N. Papanikolaou, and I. Mertig, Phys. Rev. B 73, 045428 (2006).

${ }^{29}$ S. Sanvito, C. J. Lambert, J. H. Jefferson, and A. M. Bratkovsky, Phys. Rev. B 59, 11936 (1999).

${ }^{30}$ M. Häfner, J. K. Viljas, D. Frustaglia, F. Pauly, M. Dreher, P. Nielaba, and J. C. Cuevas, Phys. Rev. B 77, 104409 (2008).

${ }^{31}$ M. Buongiorno Nardelli, Phys. Rev. B 60, 7828 (1999).

${ }^{32}$ J. Taylor, H. Guo, and J. Wang, Phys. Rev. B 63, 245407 (2001).

${ }^{33}$ M. B. Nardelli, J.-L. Fattebert, and J. Bernholc, Phys. Rev. B 64, 245423 (2001).

${ }^{34}$ Y. Xue, S. Datta, and M. A. Ratner, Chem. Phys. 281, 151 (2002).

${ }^{35}$ M. Brandbyge, J.-L. Mozos, P. Ordejón, J. Taylor, and K. Stokbro, Phys. Rev. B 65, 165401 (2002).
${ }^{36}$ K. S. Thygesen, M. V. Bollinger, and K. W. Jacobsen, Phys. Rev. B 67, 115404 (2003).

${ }^{37}$ A. Calzolari, N. Marzari, I. Souza, and M. Buongiorno Nardelli, Phys. Rev. B 69, 035108 (2004).

${ }^{38}$ S.-H. Ke, H. U. Baranger, and W. Yang, Phys. Rev. B 70, 085410 (2004).

${ }^{39}$ J. Heurich, J. C. Cuevas, W. Wenzel, and G. Schön, Phys. Rev. Lett. 88, 256803 (2002).

${ }^{40}$ P. A. Derosa and J. M. Seminario, J. Phys. Chem. B 105, 471481 (2001).

${ }^{41}$ J. J. Palacios, A. J. Pérez-Jiménez, E. Louis, and J. A. Vergés, Phys. Rev. B 64, 115411 (2001).

${ }^{42}$ J. J. Palacios, A. J. Pérez-Jiménez, E. Louis, E. SanFabián, and J. A. Vergés, Phys. Rev. B 66, 035322 (2002).

${ }^{43}$ K. Thygesen and K. Jacobsen, Chem. Phys. 319, 111 (2005).

${ }^{44}$ F. Pauly, J. Viljas, U. Huniar, M. Häfner, S. Wohlthat, M. Bürkle, J. Cuevas, and G. Schön, New J. Phys. 10, 125019 (2008).

${ }^{45}$ A. R. Rocha, V. M. García-Suárez, S. Bailey, C. Lambert, J. Ferrer, and S. Sanvito, Phys. Rev. B 73, 085414 (2006).

${ }^{46}$ A. Smogunov, A. Dal Corso, and E. Tosatti, Phys. Rev. B 70, 045417 (2004).

${ }^{47}$ A. Dal Corso, A. Smogunov, and E. Tosatti, Phys. Rev. B 74, 045429 (2006).

${ }^{48}$ N. Marzari and D. Vanderbilt, Phys. Rev. B 56, 12847 (1997).

${ }^{49}$ N.-P. Wang, Commun. Theor. Phys. 55, 158 (2011).

${ }^{50} \mathrm{M}$. Shelley, N. Poilvert, A. A. Mostofi, and N. Marzari, Comput. Phys. Commun. 182, 2174 (2011).

${ }^{51}$ D. Jacob, J. Fernández-Rossier, and J. J. Palacios, Phys. Rev. B 77, 165412 (2008)

${ }^{52}$ A. Smogunov, A. Dal Corso, and E. Tosatti, Phys. Rev. B 78, 014423 (2008).

${ }^{53}$ M. Häfner, J. K. Viljas, and J. C. Cuevas, Phys. Rev. B 79, 140410 (2009).

${ }^{54}$ Y. Mokrousov, G. Bihlmayer, and S. Blügel, Phys. Rev. B 72, 045402 (2005).

${ }^{55}$ [www.flapw.de].

${ }^{56}$ F. Freimuth, Y. Mokrousov, D. Wortmann, S. Heinze, and S. Blügel, Phys. Rev. B 78, 035120 (2008).

${ }^{57}$ J. Kuněs, R. Arita, P. Wissgott, A. Toschi, H. Ikeda, and K. Held, Comput. Phys. Commun. 181, 1888 (2010).

${ }^{58}$ S. Datta, Quantum Transport: Atom to Transistor (Cambridge University Press, Cambridge, UK, 2005).

${ }^{59}$ S. Datta, Electronic Transport in Mesoscopic Systems (Cambridge University Press, Cambridge, UK, 1995).

${ }^{60}$ F. Guinea, C. Tejedor, F. Flores, and E. Louis, Phys. Rev. B 28, 4397 (1983).

${ }^{61}$ I. Souza, N. Marzari, and D. Vanderbilt, Phys. Rev. B 65, 035109 (2001).

${ }^{62}$ G. H. Wannier, Phys. Rev. 52, 191 (1937).

${ }^{63}$ A. A. Mostofi, J. R. Yates, Y.-S. Lee, I. Souza, D. Vanderbilt, and N. Marzari, Comput. Phys. Commun. 178, 158 (2008).

${ }^{64}$ V. I. Anisimov, D. E. Kondakov, A. V. Kozhevnikov, I. A. Nekrasov, Z. V. Pchelkina, J. W. Allen, S.-K. Mo, H.-D. Kim, P. Metcalf, S. Suga et al., Phys. Rev. B 71, 125119 (2005).

${ }^{65} \mathrm{U}$. Birkenheuer and D. Izotov, Phys. Rev. B 71, 125116 (2005).

${ }^{66}$ Y.-S. Lee, M. Buongiorno Nardelli, and N. Marzari, Phys. Rev. Lett. 95, 076804 (2005). 
${ }^{67}$ A. R. Rocha, M. Rossi, A. Fazzio, and A. J. R. da Silva, Phys. Rev. Lett. 100, 176803 (2008).

${ }^{68}$ M. Shelley and A. A. Mostofi, Europhys. Lett. 94, 67001 (2011).

${ }^{69}$ N. Marzari, A. A. Mostofi, J. R. Yates, I. Souza, and D. Vanderbilt, e-print arXiv:1112.5411.

${ }^{70}$ Y. Mokrousov, G. Bihlmayer, S. Heinze, and S. Blügel, Phys. Rev. Lett. 96, 147201 (2006).
${ }^{71}$ A. Thiess, Y. Mokrousov, and S. Heinze, Phys. Rev. B 81, 054433 (2010).

${ }^{72}$ A. Smogunov, A. Dal Corso, A. Delin, R. Weht, and E. Tosatti, Nat. Nanotechnol. 3, 22 (2008).

${ }^{73}$ Y. Miura, R. Mazzarello, A. Dal Corso, A. Smogunov, and E. Tosatti, Phys. Rev. B 78, 205412 (2008).

${ }^{74}$ Y. Zhang and W. Yang, Phys. Rev. Lett. 80, 890 (1998). 